\title{
A finite difference technique for solving a time strain separable K-BKZ constitutive equation for two-dimensional moving free surface flows
}

\author{
M.F. Tomé ${ }^{\mathrm{a}, *}$, J. Bertoco ${ }^{\text {a }}$, C.M. Oishi ${ }^{\mathrm{b}}$, M.S.B. Araujo ${ }^{\mathrm{c}}$, D. Cruz $^{\mathrm{d}}$, F.T. Pinho ${ }^{\mathrm{e}}$, \\ M. Vynnycky ${ }^{\mathrm{f}}$ \\ a Departamento de Matemática Aplicada e Estatística, Universidade de São Paulo, Av. Trabalhador São-carlense, 400 - Centro, São Carlos, \\ Brazil \\ b Departamento de Matemática e Computação, Universidade Estadual Paulista “Julio de Mesquita Filho”, Rua Roberto Simonsen, 305, \\ 19060-900, Presidente Prudente, Brazil \\ c Faculdade de Matemática, Universidade Federal do Pará, Belém, Brazil \\ d Departamento de Engenharia Mecânica, Universidade Federal do Rio de Janeiro, Rio de Janeiro, Brazil \\ e Centro de Estudos de Fenómenos de Transporte, Faculdade de Engenharia da Universidade do Porto, Departamento de Engenharia Mecânica, \\ Rua Dr. Roberto Frias s/n, 4200-465, Porto, Portugal \\ ${ }^{\mathrm{f}}$ Department of Materials Science and Engineering, Royal Institute of Technology, Brinellvägen 23, 10044 Stockholm, Sweden
}

A R T I C L E I N F O

\section{Article history:}

Received 25 October 2014

Received in revised form 6 January 2016

Accepted 27 January 2016

Available online 29 January 2016

\section{Keywords:}

Integral K-BKZ constitutive equation

Deformation fields

Implicit method

Finite difference

Analytic solution in channel flow

Free surface

Jet buckling

\begin{abstract}
A B S T R A C T
This work is concerned with the numerical solution of the K-BKZ integral constitutive equation for two-dimensional time-dependent free surface flows. The numerical method proposed herein is a finite difference technique for simulating flows possessing moving surfaces that can interact with solid walls. The main characteristics of the methodology employed are: the momentum and mass conservation equations are solved by an implicit method; the pressure boundary condition on the free surface is implicitly coupled with the Poisson equation for obtaining the pressure field from mass conservation; a novel scheme for defining the past times $t^{\prime}$ is employed; the Finger tensor is calculated by the deformation fields method and is advanced in time by a second-order Runge-Kutta method. This new technique is verified by solving shear and uniaxial elongational flows. Furthermore, an analytic solution for fully developed channel flow is obtained that is employed in the verification and assessment of convergence with mesh refinement of the numerical solution. For free surface flows, the assessment of convergence with mesh refinement relies on a jet impinging on a rigid surface and a comparison of the simulation of a extrudate swell problem studied by Mitsoulis (2010) [44] was performed. Finally, the new code is used to investigate in detail the jet buckling phenomenon of K-BKZ fluids.
\end{abstract}

(C) 2016 Elsevier Inc. All rights reserved.

\section{Introduction}

Viscoelastic free surface flows are important in many industrial processes as for example in injection molding and profile extrusion. These problems are challenging as the flow may possess several moving free surfaces and although many

\footnotetext{
* Corresponding author. Tel.: +55 1633739656; fax: +55 1633739650 .

E-mail address: murilo@icmc.usp.br (M.F. Tomé).
} 
researchers have been working on the development of numerical methods for simulating viscoelastic free surface flows, the accurate application of the free surface stress conditions is a problem that has not yet been fully resolved. Most of the works and methods to deal with free surface flows of viscoelastic fluids concern constitutive equations of the differential type such as the models known as UCM [1-3], Oldroyd-B [4-7], Phan-Thien-Tanner [8-10], FENE-CR (also FENE-P) [11], eXtended Pom-Pom [12,13], among others. Notwithstanding, the advances in computational resources have motivated researchers to consider more sophisticated rheological models that employ integral equations instead of partial differential equations. One reason is that integral constitutive models are known to provide a good fitting to the rheology of polymer melts, as for example high density polyethylene (HDPE) [14,15] and low density polyethylene (LDPE) [16], that are usually employed by many industries. Among the most successful integral models stands the K-BKZ equation $[17,18]$ which has also been employed by many researchers that have developed numerical methods for this class of fluids (e.g. $[19,20]$ ). However, most of the problems investigated with integral models involve confined flows as for example entry flows $[21,22]$ and flows through abrupt contractions [16,23-25].

The K-BKZ model was originally developed using ideas of rubber elasticity theory in a general framework accounting for the strain free energy associated with the elastic strain imposed on the material $[17,18]$. In its original form, this model consists of a class of constitutive equations because it admits a large variety of functions for the free energy and it considers a viscoelastic liquid as a relaxing rubber in which both the elastic energy and stress are allowed to relax. One of the methods to arrive at a specific K-BKZ model is to fit it to single or double step strain data. The response of the model is often poor if the two sudden strains are of opposite sign [26] and this is observed not only in shear, as documented in [26], but also in extension as well shown by Rasmussen and co-workers [27,28]. These authors measured stress relaxation of some polymer melts in well-controlled extensional flow experiments, including reversed flow due to imposition of normal strain rate steps, and showed that multimode versions of models akin to the K-BKZ constitutive equation, such as the Doi-Edwards with or without interchain alignment, could not capture adequately the observed stress evolution of the melt, especially when a uniaxial extensional flow was followed by a biaxial extensional flow. In the absence of such severe strains, which is often the case, the performance of the model is significantly better and its parameters can still be determined using data from measurements of the viscosity and first normal stress difference in steady shear flow, in addition to the basic shear stress growth and shear stress relaxation experiments. If more than one mode is needed to fit the relaxation spectrum, data from small amplitude oscillatory shear provides the coefficients of the memory function.

For some polymer melts, such as LDPE, polydimethylsiloxane or polystyrene, the stress dependence of the K-BKZ equation is factorable on time and strain dependencies and the nonlinear behavior can be accommodated through a damping function, for which many forms have been proposed [29], including the Papanastasiou-Scriven-Macosko (PSM) form used here [30]. Fittings of the K-BKZ/PSM model to experimental data in steady and transient shear and extension of LDPE and HDPE are also shown in [31].

It should also be said that, as the complexity of flow kinematics increases, simple models are not able to quantitatively describe experimental data. More capable and adequate constitutive models are of integrodifferential type and are based on molecular theories for linear, branched and star polymers. These mathematically more complex models are outside the scope of the present work, but some contributions to such developments have been presented by Mead et al. [32], Ianniruberto and Marrucci [33] and Wagner et al. [34], among others.

Dealing numerically with flows of fluids described by integrodifferential models relies most commonly on extensions of Lagrangian approaches, such as the Lagrangian integral method of Hassager and Bisgaard [35], subsequently extended to 3D flows by Rasmussen and Hassager [36], or with extensions of the method of deformation fields of Peters et al. [37] used here also. One example of an extension of Lagrangian methods is by Peters el al. [38], whereas an early extension of the Eulerian method is by Wapperom et al. [39] who performed simulations in a sudden contraction-expansion.

Flow problems possessing free surface(s) have also been considered by some investigators. For example, one work involving transient free surface flows and integral models is the filament stretching presented by McKinley and Sridhar [40] and was also studied in $[36,41,42]$. Another free surface flow problem which has been numerically investigated is the extrudate swell phenomenon [14,43-52]. However, these investigations employ the finite element method and their application to flows possessing time-dependent folding free surfaces has yet to be demonstrated. A detailed discussion on the importance of the K-BKZ integral constitutive model and current developments on numerical techniques for solving integral models are provided in the review papers of Tanner [53] and Mitsoulis [31]. For this reason, a more general introduction on numerical methods for solving integral constitutive equations is not given here.

Free surface flows in which there is a moving free surface interacting with solid walls are common in practical applications such as container filling. Even though the K-BKZ model has been applied to solve a variety of viscoelastic flows, to the best of our knowledge, integral constitutive models have not been applied to simulate the buckling effect that can occur when a jet interacts with a solid wall. It is true that such interaction results in a combination of uniaxial and biaxial extensional flows for which the K-BKZ, and several other simple integral constitutive equations [27,28], cannot provide an accurate quantitative solution, but as indicated above and below, there is sufficient novelty in the set of characteristics of this work to justify this numerical experience. Although there are various versions of the K-BKZ integral model, this work deals with the so-called K-BKZ/PSM model (Papanastasiou-Scriven-Macosko [30]).

We present a numerical method for solving two-dimensional flows governed by the K-BKZ/PSM integral constitutive equation that is capable of resolving multiple moving free surface flows and their interaction with rigid walls. We formulate 
an algorithm to resolve the governing equations and employ the finite difference method to solve the basic equations. The developed numerical method is extensively verified by several analytic and numerical solutions as follows:

1. Shear and elongational flows are solved analytically and numerically, with the former used to verify the latter.

2. An analytic solution for the fully developed channel flow is presented and again, it is used to verify the computed numerical solution of 2D channel flow.

3. To demonstrate convergence of the methodology on moving free surface flows for integral constitutive equations, a jet impinging on a stationary flat plate is simulated on six consecutively refined meshes. Several snapshots taken at selected times are plotted and the results display convergence of the free surface with mesh refinement. Moreover, the extrudate swell problem was simulated and the results obtained were compared to the solution presented by Mitsoulis [44].

\section{Governing equations}

The governing equations for isothermal incompressible flows are the mass conservation equation and the equation of motion which, in dimensionless form, can be written as (for details, see Tomé et al. [25])

$$
\begin{aligned}
\nabla \cdot \mathbf{v} & =0, \\
\frac{\partial \mathbf{v}}{\partial t}+\nabla \cdot(\mathbf{v v}) & =-\nabla p+\frac{1}{R e} \nabla^{2} \mathbf{v}+\nabla \cdot \boldsymbol{\Phi}+\frac{1}{F r^{2}} \mathbf{g} .
\end{aligned}
$$

In equation (2), $\boldsymbol{\Phi}$ is a non-Newtonian stress tensor that is related to the extra-stress tensor through the following EVSS transformation [54]:

$$
\boldsymbol{\Phi}=\boldsymbol{\tau}-\frac{1}{R e} \dot{\gamma}, \text { where } \dot{\gamma}=\nabla \mathbf{v}+(\nabla \mathbf{v})^{\mathrm{T}} .
$$

These equations must be complemented by considering a rheological model that defines the behavior of fluid flow. In this work, the K-BKZ/PSM [30] integral constitutive equation is adopted, namely

$$
\boldsymbol{\tau}(t)=\int_{-\infty}^{t} M\left(t-t^{\prime}\right) H\left(I_{1}, I_{2}\right) \mathbf{B}_{t^{\prime}}(t) d t^{\prime}
$$

where

$$
M\left(t-t^{\prime}\right)=\sum_{k=1}^{m_{1}} \frac{a_{k}}{\lambda_{k} W i} e^{-\frac{t-t^{\prime}}{\lambda_{k} W i}}
$$

is the memory function, $\lambda_{k}, a_{k}, m_{1}$ are relaxation times, relaxation moduli and the number of relaxation modes, respectively. The function

$$
H\left(I_{1}, I_{2}\right)=\frac{\alpha}{\alpha-3+\beta I_{1}+(1-\beta) I_{2}}
$$

is the Papanastasiou-Scriven-Macosko [30] damping function and $\mathbf{B}_{t^{\prime}}(t)$ is the Finger tensor. The quantities $I_{1}=\operatorname{tr}\left[\mathbf{B}_{t^{\prime}}(t)\right.$ ] and $I_{2}=\frac{1}{2}\left(\left(I_{1}\right)^{2}-\operatorname{tr}\left[\mathbf{B}_{t^{\prime}}^{2}(t)\right]\right)$, are the first and second invariants of $\mathbf{B}_{t^{\prime}}(t)$, respectively. The parameters $a_{k}, \lambda_{k}, \alpha, \beta$ are obtained from a curve fitting to the rheological properties of the fluid.

In these equations, $\operatorname{Re}=\frac{\rho_{0} U L}{\eta_{0}}$ is the Reynolds number, $F r=\frac{U}{\sqrt{L g}}$ is the Froude number and $W i=\lambda_{\text {ref }} \frac{U}{L}$ is the Weissenberg number, where $U$ and $L$ are velocity and length scales, $g$ and $\rho_{0}$ are the gravity acceleration and fluid density, respectively. Additionally, $\eta_{0}=\sum_{k=1}^{m_{1}} a_{k} \lambda_{k}$ is the zero-shear-rate viscosity and $\lambda_{\text {ref }}=\sum \frac{a_{k} \lambda^{2} k}{a_{k} \lambda_{k}}$ is the average relaxation time [44]. The nondimensionalization of the equations was performed by using the following variables (the dimensional variables are denoted by an overbar):

$$
\overline{\mathbf{x}}=L \mathbf{x}, \bar{t}=\frac{L}{U} t, \overline{\mathbf{v}}=U \mathbf{v}, \quad \bar{p}=\rho_{0} U^{2} p, \quad \overline{\boldsymbol{\tau}}=\rho_{0} U^{2} \boldsymbol{\tau}, \quad \bar{\lambda}_{k}=\lambda_{\text {ref }} \lambda_{k}, \quad \bar{a}_{k}=\rho_{0} U^{2} a_{k} .
$$

Equations (1)-(3) are solved for two-dimensional Cartesian time-dependent flows where $p=p(x, y, t), \mathbf{v}=(u(x, y, t)$, $v(x, y, t))^{T}$ and

$$
\boldsymbol{\tau}(x, y, t)=\left[\begin{array}{cc}
\tau^{x x} & \tau^{x y} \\
\tau^{x y} & \tau^{y y}
\end{array}\right], \quad \boldsymbol{\Phi}(x, y, t)=\left[\begin{array}{cc}
\Phi^{x x} & \Phi^{x y} \\
\Phi^{x y} & \Phi^{y y}
\end{array}\right], \quad \mathbf{B}_{t^{\prime}(t)}(x, y, t)=\left[\begin{array}{ccc}
B^{x x} & B^{x y} & 0 \\
B^{x y} & B^{y y} & 0 \\
0 & 0 & 1
\end{array}\right] .
$$

Note that to compute the stresses for 2D flows we need to consider the 3D Finger tensor as shown. 


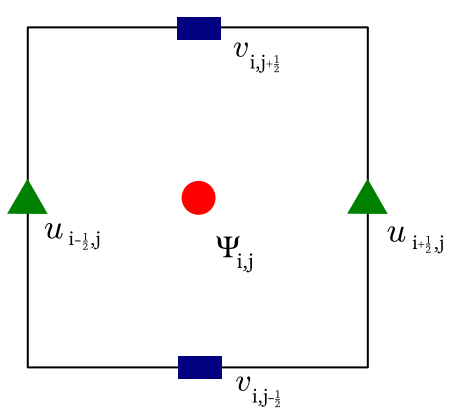

(a)

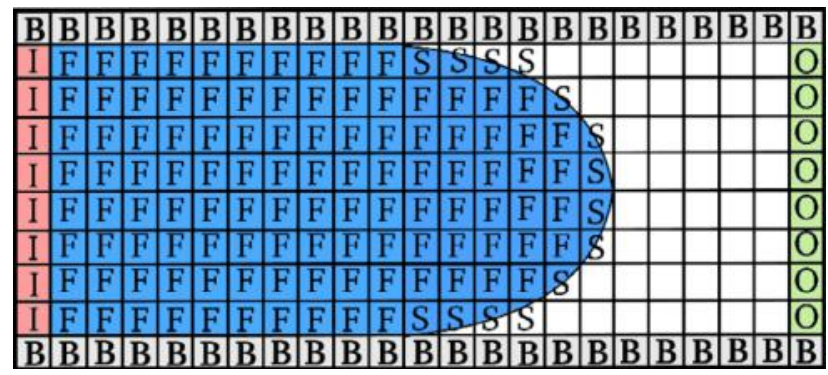

(b)

Fig. 1. (a) Typical two-dimensional staggered cell. (b) Illustration of cell type classification used.

\subsection{Boundary conditions}

To solve equations (1)-(3), appropriate boundary conditions for the velocity field must be specified. At fluid entries (inflows) the velocity ( $\mathbf{V}_{\text {inf }}$ ) is prescribed while at fluid exits (outflows) fully developed flow is assumed and so the homogeneous Neumann condition, $\frac{\partial \mathbf{v}}{\partial n}=\mathbf{0}$, is applied, where $n$ denotes the normal direction to the boundary. The details on the specification of the velocity at inflows are provided in the sections dealing with numerical results.

On fluid surfaces it is necessary to apply special boundary conditions for the velocity and pressure fields. In this work we consider unsteady free surface flows of fluids moving into a passive atmosphere. In the absence of surface tension forces, the normal and tangential components of stress must be continuous across any surface, so that on the fluid free surface the following conditions must hold (see [55])

$$
\mathbf{n} \cdot \boldsymbol{\sigma} \cdot \mathbf{n}^{\mathrm{T}}=0 \quad \text { and } \quad \mathbf{m} \cdot \boldsymbol{\sigma} \cdot \mathbf{n}^{\mathrm{T}}=0,
$$

where $\mathbf{n}$ is the unit outward normal vector to the free surface and $\mathbf{m}$ is the associated unit tangential vector. The total stress tensor, $\sigma$ is given by

$$
\sigma=-p \mathbf{I}+\frac{1}{R e} \dot{\gamma}+\boldsymbol{\Phi},
$$

where $\Phi$ and $\dot{\gamma}$ are the tensors obtained from the application of the EVSS transformation (3). For two-dimensional Cartesian flows, the unit vectors $\mathbf{n}$ and $\mathbf{m}$ in the stress conditions (8) can be taken as $\mathbf{n}=\left(n_{x}, n_{y}\right)$ and $\mathbf{m}=\left(n_{y},-n_{x}\right)$, in which case these conditions can be written simply as

$$
p=\frac{2}{R e}\left[\frac{\partial u}{\partial x} n_{x}^{2}+\left(\frac{\partial u}{\partial y}+\frac{\partial v}{\partial x}\right) n_{x} n_{y}+\frac{\partial v}{\partial y} n_{y}^{2}\right]+\Phi^{x x} n_{x}^{2}+2 \Phi^{x y} n_{x} n_{y}+\Phi^{y y} n_{y}^{2}
$$

and

$$
\frac{1}{\operatorname{Re}}\left[2\left(\frac{\partial u}{\partial x}-\frac{\partial v}{\partial y}\right) n_{x} n_{y}+\left(\frac{\partial u}{\partial y}+\frac{\partial v}{\partial x}\right)\left(n_{y}^{2}-n_{x}^{2}\right)\right]+\left(\Phi^{x x}-\Phi^{y y}\right) n_{x} n_{y}+\Phi^{x y}\left(n_{y}^{2}-n_{x}^{2}\right)=0 .
$$

\section{Numerical method}

The governing equations are solved by a variant of the original Marker-And-Cell method [56,57] that employs the finite difference method on a staggered grid. The velocity components are located at the middle of cell faces while the other variables, denoted by $\boldsymbol{\Psi}$, are positioned at cell centres (see Fig. 1(a)). We consider flows possessing moving free surfaces so that a strategy to follow the free surface and to define the fluid region is employed as represented in Fig. 1(b). This work applies the technique presented by Tomé et al. [58,59], in which the fluid surface is composed by marker particles that move with the local fluid velocity. The visualization of the fluid surface (also the free surface) is affected by connecting the particles by straight lines (see Fig. 2(a)). The body of the fluid is represented by the region within the closed curve defined by connecting the particles (see Fig. 2(b)). This strategy requires that the cells within the mesh are arranged in the groups defined below (see [59] and Fig. 1(b)):

Inflow (I): cells that define fluid entrances;

Outflow (0): cells that define fluid exits;

- Boundary (B): cells that define rigid boundaries;

- Empty (E): cells that do not contain fluid and are represented by blank cells in Fig. 1(b); 


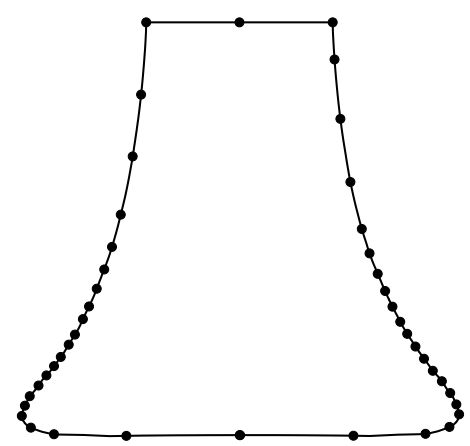

(a) Definition of the fluid surface.

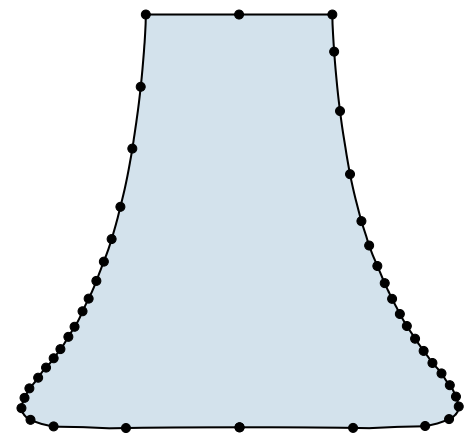

(b) Visualization of the volume of fluid.

Fig. 2. Representation of the fluid.

Surface (S): cells that contain fluid and have one or more faces in contact with E-cell faces.

Full (F): cells that define the fluid region. These cells are required not to have any face in contact with $\mathbf{E}$-cell faces.

The solutions $\mathbf{v}\left(\mathbf{x}, t_{n+1}\right), p\left(\mathbf{x}, t_{n+1}\right)$ and $\boldsymbol{\tau}\left(\mathbf{x}, t_{n+1}\right)$ at time $t_{n+1}=t+\delta t$ are obtained in two parts: first, using the values of $\boldsymbol{\tau}(\mathbf{x}, t)$, the velocity and pressure fields are calculated at time $t_{n+1}$. Then, $\mathbf{v}\left(\mathbf{x}, t_{n+1}\right)$ is employed to compute the tensor $\boldsymbol{\tau}\left(\mathbf{x}, t_{n+1}\right)$ by the deformation fields method [60], as follows.

\subsection{Calculation of $\mathbf{v}\left(\mathbf{x}, t_{n+1}\right)$ and $p\left(\mathbf{x}, t_{n+1}\right)$}

The numerical method employed to solve the mass (1) and momentum equations (2) is based on the implicit algorithm proposed by Oishi et al. [13] for solving viscoelastic free surface flows of an eXtended Pom-Pom fluid model, as follows.

It is assumed that at time $t$ the variables $\mathbf{v}(\mathbf{x}, t)=\mathbf{v}^{(n)}, p(\mathbf{x}, t)=p^{(n)}, \boldsymbol{\tau}(\mathbf{x}, t)=\boldsymbol{\tau}^{(n)}$ and the markers' positions $\mathbf{x}(t)=\mathbf{x}^{(n)}$, are known. Then, $\mathbf{v}\left(\mathbf{x}, t_{n+1}\right), p\left(\mathbf{x}, t_{n+1}\right)$ and $\mathbf{x}\left(t_{n+1}\right)$ are obtained as follows:

1. Calculate $\dot{\boldsymbol{\gamma}}^{(n)}=\left[\nabla \mathbf{v}^{(n)}+\left(\nabla \mathbf{v}^{(n)}\right)^{\mathrm{T}}\right]$ and from (3) obtain $\boldsymbol{\Phi}=\boldsymbol{\tau}^{(n)}-\frac{1}{\operatorname{Re}} \dot{\boldsymbol{\gamma}}^{(n)}$.

2. Calculate an intermediate velocity field $\widetilde{\mathbf{v}}^{(n+1)}$.

By using the ideas of the projection method (see [13]) to uncouple the mass and momentum equations, an intermediate velocity field $\widetilde{\mathbf{v}}^{(n+1)}$ is obtained from Eq. (2). In this work we employ the Crank-Nicolson discretization for the diffusion term to calculate $\widetilde{\mathbf{v}}^{(n+1)}$, i.e.,

$$
\frac{\widetilde{\mathbf{v}}^{(n+1)}}{\delta t}-\frac{1}{2 \operatorname{Re}} \nabla^{2} \widetilde{\mathbf{v}}^{(n+1)}=\frac{\mathbf{v}^{(n)}}{\delta t}-\nabla \cdot(\mathbf{v v})^{(n)}-\nabla p^{(n)}+\frac{1}{2 \operatorname{Re}} \nabla^{2} \mathbf{v}^{(n)}+\nabla \cdot \boldsymbol{\Phi}+\frac{1}{F r^{2}} \mathbf{g},
$$

where the boundary conditions for $\widetilde{\mathbf{v}}^{(n+1)}$ are the same as those for the final velocity $\mathbf{v}^{(n+1)}$, to be determined below, and $p^{(n)}$ is an approximation to $p^{(n+1)}$. It can be shown that $\widetilde{\mathbf{v}}^{(n+1)}$ possesses the correct vorticity at time $t_{n+1}$ but it does not conserve mass in general [61]. Therefore, there is a potential function $\psi^{(n+1)}$ so that

$$
\mathbf{v}^{(n+1)}=\widetilde{\mathbf{v}}^{(n+1)}-\nabla \psi^{(n+1)}
$$

where by imposing mass conservation for $\mathbf{v}^{(n+1)}$, the function $\psi^{(n+1)}$ must satisfy the Poisson equation (14). This intermediate velocity field is obtained for all $\mathbf{F}$ - and $\mathbf{S}$-cells within the domain. For this, two positive definite linear systems are solved efficiently by the conjugate gradient method.

3. Solve the Poisson equation for the potential function $\psi$ :

$$
\nabla^{2} \psi^{(n+1)}=\nabla \cdot \widetilde{\mathbf{v}}^{(n+1)} .
$$

The boundary conditions required for solving this Poisson equation are the homogeneous Neumann conditions for rigid walls and inflows, while homogeneous Dirichlet conditions are applied at outflows. This equation is solved for every F-cell in the domain.

However, to maintain the stability of the numerical scheme when solving low Reynolds number free surface flows, the implicit formulation proposed in [13] that employs equations for $\psi^{(n+1)}$ on the free surface, is used here. Oishi et al. [13] made use of the pressure condition on the free surface (10) and derived new equations for $\psi^{(n+1)}$ on $\mathbf{S}$-cells that can be cast in the following equation: 


$$
\begin{aligned}
& \frac{\psi^{(n+1)}}{\delta t}-\frac{2}{\operatorname{Re}}\left[\left(\frac{\partial^{2} \psi^{(n+1)}}{\partial y^{2}}\right) n_{x}^{2}+\left(\frac{\partial^{2} \psi^{(n+1)}}{\partial x^{2}}\right) n_{y}^{2}-2\left(\frac{\partial^{2} \psi^{(n+1)}}{\partial x \partial y}\right) n_{x} n_{y}\right] \\
& =\frac{2}{\operatorname{Re}}\left[-\left(\frac{\partial \widetilde{v}^{(n+1)}}{\partial y}\right) n_{x}^{2}-\left(\frac{\partial \widetilde{u}^{(n+1)}}{\partial x}\right) n_{y}^{2}+\left(\frac{\partial \widetilde{u}^{(n+1)}}{\partial y}+\frac{\partial \widetilde{v}^{(n+1)}}{\partial x}\right) n_{x} n_{y}\right] \\
& \quad+\left[\Phi^{x x} n_{x}^{2}+2 \Phi^{x y} n_{x} n_{y}+\Phi^{y y} n_{y}^{2}\right]-p^{(n)} .
\end{aligned}
$$

This equation is applied to every surface cell $(\mathbf{S})$ according to local free surface orientations that can be obtained by analyzing empty-cell neighbors (E), namely:

- Planar surface: if the $\mathbf{S}$-cell has only one face in contact with an $\mathbf{E}$-cell face then the free surface is assumed to be either vertical or horizontal, in which case the normal vector $\mathbf{n}$ takes the form $(0, \pm 1)^{T}$ or $( \pm 1,0)^{T}$. For instance, if $\mathbf{n}=(1,0)^{T}$ then equation $(15)$ reduces to

$$
\frac{\psi^{(n+1)}}{\delta t}-\frac{2}{\operatorname{Re}} \frac{\partial^{2} \psi^{(n+1)}}{\partial y^{2}}=-\frac{2}{\operatorname{Re}} \frac{\partial \widetilde{v}^{(n+1)}}{\partial y}+\Phi^{x x}-p^{(n)} .
$$

- $45^{\circ}$ sloped linear surface: If the $\mathbf{S}$-cell has only two adjacent faces that are contiguous with $\mathbf{E}$-cell faces then the free surface is taken to be linear making an angle of $45^{\circ}$ with the coordinate axes. In this case, the normal vector can be described by $\mathbf{n}=\left( \pm \frac{\sqrt{2}}{2}, \pm \frac{\sqrt{2}}{2}\right)$. For example, when $\mathbf{n}=\left(\frac{\sqrt{2}}{2}, \frac{\sqrt{2}}{2}\right)$, after enforcement of mass conservation, equation (15) is reduced to

$$
\frac{\psi^{(n+1)}}{\delta t}+\frac{2}{R e} \frac{\partial^{2} \psi^{(n+1)}}{\partial x \partial y}=\frac{1}{R e}\left[\frac{\partial \widetilde{u}^{(n+1)}}{\partial y}+\frac{\partial \widetilde{v}^{(n+1)}}{\partial x}\right]+\frac{1}{2}\left[\Phi^{x x}+2 \Phi^{x y}+\Phi^{y y}\right]-p^{(n)} .
$$

For surface cells that have two opposite faces that are in contact with E-cell faces, an approximate expression for the normal vector cannot be attributed. For these cells, the potential function $\psi^{(n+1)}$ is assumed to be zero.

The combination of Eq. (14) in F-cells and Eq. (15) applied to S-cells leads to a large non-symmetric linear system which is solved efficiently by the Bi-conjugate gradient method (cf. [13]).

We point out that the tangential stress condition given by Eq. (11) is equal to the corresponding equation used when solving free surface flows of a Phan-Thien-Tanner fluid (see [9]). It is treated in the same way as in Paulo et al. [9] and its approximation is not given here.

4. Compute the final velocity field from equation (13).

5. Compute the final pressure field by [61]:

$$
p^{(n+1)}=p^{(n)}+\frac{\psi^{(n+1)}}{\delta t} .
$$

The equations in steps 1 to 5 are discretized using a second-order finite differences, although first-order finite differences are used to approximate the boundary conditions. The corresponding approximated finite-difference forms of these equations have been described in detail in the works of Oishi et al. [12,13] and for this reason they are not presented here.

\subsection{Calculation of the extra-stress tensor $\boldsymbol{\tau}\left(\mathbf{x}, t_{n+1}\right)$}

To calculate the extra-stress tensor $\boldsymbol{\tau}\left(\mathbf{x}, t_{n+1}\right)$ we make use of the methodology presented by Tomé et al. [25] as follows. Let $t_{j}^{\prime}, j=0,1, \cdots, N$, be $(N+1)$-points in the interval $\left[0, t_{n+1}\right]$. Then the constitutive equation (4) can be written in the form

$$
\begin{aligned}
\boldsymbol{\tau}\left(t_{n+1}\right)= & \int_{-\infty}^{0} M\left(t_{n+1}-t^{\prime}\right) H\left(I_{1}, I_{2}\right) \mathbf{B}_{t^{\prime}}\left(t_{n+1}\right) d t^{\prime} \\
& +\sum_{j=0}^{\frac{N-2}{2}} \int_{t_{2 j}^{\prime}}^{t_{2 j+2}^{\prime}} M\left(t_{n+1}-t^{\prime}\right) H\left(I_{1}, I_{2}\right) \mathbf{B}_{t^{\prime}}\left(t_{n+1}\right) d t^{\prime},
\end{aligned}
$$

where $N$ even is adopted. For $t^{\prime}<0, \mathbf{B}_{t^{\prime}}\left(t_{n+1}\right)=\mathbf{B}_{0}\left(t_{n+1}\right)$ and therefore, the first integral becomes

$$
\int_{-\infty}^{0} M\left(t_{n+1}\right) H\left(I_{1}\left(\mathbf{B}_{0}\left(t_{n+1}\right)\right), I_{2}\left(\mathbf{B}_{0}\left(t_{n+1}\right)\right)\right) \mathbf{B}_{0}\left(t_{n+1}\right) d t^{\prime}
$$

and can be solved exactly. With regard to the integrals under the summation operator in equation (19), if $t_{j}^{\prime}$ are equally spaced then each integral can be solved by the third order Simpson's rule. However, in general, the points $t_{j}^{\prime}$ are not equally 
spaced and a second-order quadrature formula using the method of undetermined coefficients is employed. In this work, each integral $\int_{t_{2 j}^{\prime}}^{t_{2 j+2}^{\prime}} M\left(t_{n+1}-t^{\prime}\right) H\left(I_{1}, I_{2}\right) \mathbf{B}_{t^{\prime}}\left(t_{n+1}\right) d t^{\prime}$ is approximated by the 3-node quadrature formula

$$
\begin{aligned}
I_{3}= & A_{0} * H\left(I_{1}\left(\mathbf{B}_{t_{2 j}^{\prime}}\left(t_{n+1}\right)\right), I_{2}\left(\mathbf{B}_{t_{2 j}^{\prime}}\left(t_{n+1}\right)\right)\right) \mathbf{B}_{t_{2 j}^{\prime}}\left(t_{n+1}\right) \\
& +A_{1} * H\left(I_{1}\left(\mathbf{B}_{t_{2 j+1}^{\prime}}\left(t_{n+1}\right)\right), I_{2}\left(\mathbf{B}_{t_{2 j+1}^{\prime}}\left(t_{n+1}\right)\right)\right) \mathbf{B}_{t_{2 j+1}^{\prime}}\left(t_{n+1}\right) \\
& +A_{2} * H\left(I_{1}\left(\mathbf{B}_{t_{2 j+2}^{\prime}}\left(t_{n+1}\right)\right), I_{2}\left(\mathbf{B}_{t_{2 j+2}^{\prime}}\left(t_{n+1}\right)\right)\right) \mathbf{B}_{t_{2 j+2}^{\prime}}\left(t_{n+1}\right) .
\end{aligned}
$$

The coefficients $A_{0}, A_{1}, A_{2}$ are obtained by solving the $(3 \times 3)$-linear system

$$
\begin{aligned}
A_{0}+A_{1}+A_{2}=b_{0} & =\int_{t_{2 j}^{\prime}}^{t_{2 j+2}^{\prime}} M\left(t_{n+1}-t^{\prime}\right) d t^{\prime}, \\
A_{0} * t_{2 j}^{\prime}+A_{1} * t_{2 j+1}^{\prime}+A_{2} * t_{2 j+2}^{\prime} & =b_{1}=\int_{t_{2 j}^{\prime}}^{t_{2 j+2}^{\prime}} M\left(t_{n+1}-t^{\prime}\right) t^{\prime} d t^{\prime}, \\
A_{0} *\left(t_{2 j}^{\prime}\right)^{2}+A_{1} *\left(t_{2 j+1}^{\prime}\right)^{2}+A_{2} *\left(t_{2 j+2}^{\prime}\right)^{2} & =b_{2}=\int_{t_{2 j}^{\prime}}^{t_{2 j+2}^{\prime}} M\left(t_{n+1}-t^{\prime}\right)\left(t^{\prime}\right)^{2} d t^{\prime},
\end{aligned}
$$

and are found to be

$$
\begin{aligned}
& A_{2}=\frac{t_{2 j+1}^{\prime} t_{2 j}^{\prime} b_{0}+b_{2}-t_{2 j}^{\prime} b_{1}-t_{2 j+1}^{\prime} b_{1}}{\left(t_{2 j+2}^{\prime}\right)^{2}-t_{2 j+1}^{\prime} t_{2 j+2}^{\prime}+t_{2 j+1}^{\prime} t_{2 j}^{\prime}-t_{2 j}^{\prime} t_{2 j+2}^{\prime}}, \\
& A_{1}=\frac{-t_{2 j}^{\prime} b_{1}+t_{2 j}^{\prime} b_{0} t_{2 j+2}^{\prime}+b_{2}-t_{2 j+2}^{\prime} b_{1}}{\left(t_{2 j+1}^{\prime}-t_{2 j}^{\prime}\right)\left(t_{2 j+1}^{\prime}-t_{2 j+2}^{\prime}\right)}, \\
& A_{0}=-\frac{-t_{2 j+1}^{\prime} b_{1}+t_{2 j+1}^{\prime} b_{0} t_{2 j+2}^{\prime}+b_{2}-t_{2 j+2}^{\prime} b_{1}}{\left(-t_{2 j+2}^{\prime}+t_{2 j}^{\prime}\right)\left(t_{2 j+1}^{\prime}-t_{2 j}^{\prime}\right)} .
\end{aligned}
$$

In the following two sections, details on how the points $t_{j}^{\prime}\left(t_{n+1}\right)$ are calculated and the computation of the Finger tensor $\mathbf{B}_{t^{\prime}\left(t_{n+1}\right)}\left(t_{n+1}\right)$ are presented.

\subsubsection{Discretization of the time interval $\left[0, t_{n+1}\right]$}

One of the key issues of the deformation fields method is how the integration nodes $0 \leq t_{0}^{\prime}<t_{1}^{\prime}<\cdots<t_{N}^{\prime}=t_{n+1}$ are distributed over the interval $\left[0, t_{n+1}\right]$, because such distribution can affect the accuracy of the results when solving complex flows. For instance, Hulsen et al. [60] used the concepts of age time, $\mathcal{S}=t-t^{\prime}$, and cutoff age, $\mathcal{S}_{c}$, and employed a stretched mesh, with finer time steps close to the present time $t_{n+1}$, over the interval $\left[0, \mathcal{S}_{c}\right]$ to compute $\boldsymbol{\tau}\left(\mathbf{x}, t_{n+1}\right)$ and simulated the steady flow over a sphere using both UCM and KBKZ/PSM models. However, how to apply their methodology to timedependent free surface flows is unclear. In the work of Tomé et al. [25], the memory function is used to split the interval $\left[0, t_{n+1}\right]$ into $N$-subintervals $\left[t_{j-1}^{\prime}, t_{j}^{\prime}\right], j=1,2, \cdots, N$, with $t_{0}^{\prime}=0, t_{N}^{\prime}=t_{n+1}$ and $t_{j}^{\prime}$ is given by

$$
t_{j}^{\prime}=\frac{\sum_{k=1}^{m_{1}} t_{j_{k}}^{\prime}}{m_{1}}, j=1,2, \cdots, N,
$$

where $t_{j_{k}}^{\prime}$ are calculated by

$$
t_{j_{k}}^{\prime}\left(t_{n+1}\right)=\lambda_{k} W i \ln \left(\frac{\lambda_{k} W i}{a_{k}} M_{j_{k}}\left(t_{n+1}\right)\right)+t, \quad k=1,2, \cdots, m_{1}
$$

where $M_{j_{k}}\left(t_{n+1}\right)=M_{k}\left(t_{n+1}\right)+j \cdot d M_{k}, j=1,2, \cdots, N$ and $d M_{k}=\frac{M_{k}(0)-M_{k}\left(t_{n+1}\right)}{N}$ pertains to mode $k$. This also provides variable time steps, with finer steps closer to the present, and related to the time responses of the fluid.

Tomé et al. [25] obtained results for channel and contraction flows, and in their error analysis they showed that this methodology for obtaining $t_{j}^{\prime}\left(t_{n+1}\right)$ worked well for shear flows, but not for elongational flows.

In this work we present an alternative ad hoc methodology for the discretization of the interval [0, $\left.t_{n+1}\right]$. Instead of using the inverse image of the memory function, a geometric progression is employed to calculate the integration nodes. We consider time-dependent flows so that the integration nodes are calculated at time $t_{n+1}$ as follows: 


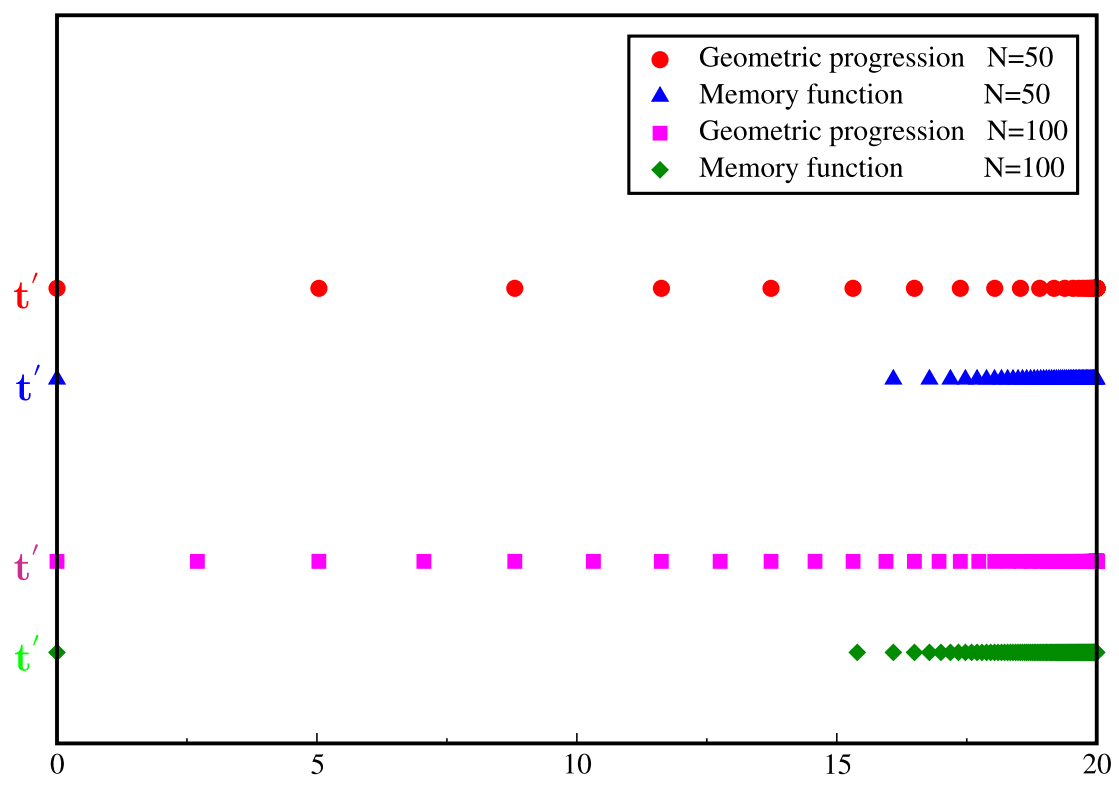

Fig. 3. Discretization of the interval $\{0,20\}$ into 50 and 100 points using a geometric progression and the inverse image of the memory function.

1. Set $t_{0}^{\prime}=0$ and $t_{N}^{\prime}=t_{n+1}$;

2. $t_{N-j}^{\prime}=t_{N}^{\prime}-q^{j}, j=1,2, \cdots, N-1$, where $q=\left(t_{n+1} / \delta t\right)^{1 / N}, d t$ is the time-step employed for calculating the velocity and pressure (see Section 3.1).

To illustrate the application of this new scheme in the discretization of the interval $\{0,20\}$, Fig. 3 shows the distribution of $t_{j}^{\prime}\left(t_{n+1}\right)$ obtained by this geometric progression using $N=50,100$ and compares it with the distribution from the single mode memory function $M\left(t_{n+1}-t^{\prime}\left(t_{n+1}\right)\right)=100 e^{-\left(t_{n+1}-t^{\prime}\left(t_{n+1}\right)\right)}$ used previously. As we can see, in the discretizations of the interval $\{0,20\}$ by the memory function, the points $t_{j}^{\prime}\left(t_{n+1}\right)$ lie in the range $\{15,20\}$ whereas the whole range is covered by the discretization using the geometric progression. The effect of the discretization on the accuracy of the computations is discussed in the section of validation results.

\subsubsection{Computation of the Finger tensor $\mathbf{B}_{t^{\prime}\left(t_{n+1}\right)}\left(\mathbf{x}, t_{n+1}\right)$}

One of the difficulties in the numerical simulation of viscoelastic flows using integral constitutive models is how to calculate the strain history accurately. In finite elements this can be accomplished by a particle-tracking method based on the velocity field (see [21]), but here a different approach is employed. We follow on the ideas of the deformation fields method [60] in which the Finger tensor is computed by solving an appropriate evolution equation where $\mathbf{B}_{t^{\prime}(t)}(\mathbf{x}, t)$ is given by

$$
\frac{\partial}{\partial t} \mathbf{B}_{t^{\prime}(t)}(\mathbf{x}, t)+\nabla \cdot\left(\mathbf{v}(\mathbf{x}, t) \mathbf{B}_{t^{\prime}(t)}(\mathbf{x}, t)\right)=[\nabla \mathbf{v}(\mathbf{x}, t)]^{T} \cdot \mathbf{B}_{t^{\prime}(t)}(\mathbf{x}, t)+\mathbf{B}_{t^{\prime}(t)}(\mathbf{x}, t) \cdot \nabla \mathbf{v}(\mathbf{x}, t),
$$

with the condition $\mathbf{B}_{t^{\prime}=t_{n+1}}\left(\mathbf{x}, t_{n+1}\right)=\mathbf{I}$. To solve (30) in time we first rewrite it in the form

$$
\frac{\partial}{\partial t} \mathbf{B}_{t^{\prime}(t)}(\mathbf{x}, t)=\mathcal{G}[\mathbf{v}, \mathbf{B}]
$$

where

$$
\mathcal{G}[\mathbf{v}, \mathbf{B}]=-\nabla \cdot\left(\mathbf{v}(\mathbf{x}, t) \mathbf{B}_{t^{\prime}(t)}(\mathbf{x}, t)\right)+\left[(\nabla \mathbf{v}(\mathbf{x}, t))^{T} \cdot \mathbf{B}_{t^{\prime}(t)}(\mathbf{x}, t)+\mathbf{B}_{t^{\prime}(t)}(\mathbf{x}, t) \cdot \nabla \mathbf{v}(\mathbf{x}, t)\right] .
$$

The Finger tensor $\mathbf{B}_{t^{\prime}(t)}\left(\mathbf{x}, t_{n+1}\right)$ is calculated by the second-order modified Euler method, namely

$$
\mathbf{B}_{t^{\prime}(t)}\left(\mathbf{x}, t_{n+1}\right)=\mathbf{B}_{t^{\prime}(t)}(\mathbf{x}, t)+\frac{\delta t}{2}\left\{\mathcal{G}\left[\mathbf{v}^{(n+1)}, \mathbf{B}_{t^{\prime}(t)}(\mathbf{x}, t)\right]+\mathcal{G}\left[\mathbf{v}^{(n+1)}, \overline{\mathbf{B}}_{t^{\prime}(t)}\left(\mathbf{x}, t_{n+1}\right)\right]\right\},
$$

where

$$
\overline{\mathbf{B}}_{t^{\prime}(t)}\left(\mathbf{x}, t_{n+1}\right)=\mathbf{B}_{t^{\prime}(t)}(\mathbf{x}, t)+\delta t \mathcal{G}\left[\mathbf{v}^{(n+1)}, \mathbf{B}_{t^{\prime}(t)}(\mathbf{x}, t)\right] .
$$

When computing the convective term of (32) in F-cells, the high order upwind scheme CUBISTA [62] is employed. This requires the values of the Finger tensor on mesh boundaries (cells B, I, O), as is explained in detail by Tomé et al. [25]. We 


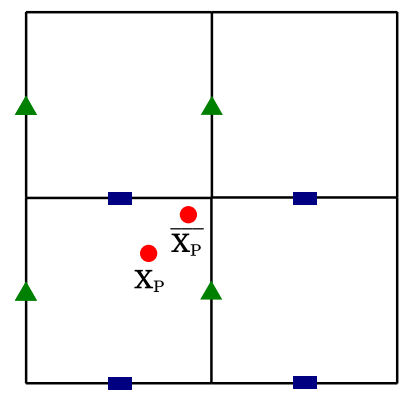

Fig. 4. Velocities employed when calculating the velocity of particle $\mathbf{v}\left(\mathbf{x}_{P}(t), t_{n+1}\right)=\left(u\left(\mathbf{x}_{P}(t), t_{n+1}\right), v\left(\mathbf{x}_{P}(t), t_{n+1}\right)\right)$ by bilinear interpolation. The velocity $u\left(\mathbf{x}_{P}(t), t_{n+1}\right)$ is obtained using the velocities represented by the green symbols $(\boldsymbol{\Delta})$ while the calculation of $v\left(\mathbf{x}_{P}(t), t_{n+1}\right)$ involves the velocities represented by the blue symbols ( $\boldsymbol{\square}$ ). For details, see [58]. (For interpretation of the references to color in this figure legend, the reader is referred to the web version of this article.)

point out that the Finger tensor $\mathbf{B}_{t^{\prime}(t)}\left(\mathbf{x}, t_{n+1}\right)$ is calculated at the past times $t^{\prime}(t)$. The updated Finger tensor $\mathbf{B}_{t^{\prime}\left(t_{n+1}\right)}\left(\mathbf{x}, t_{n+1}\right)$ is evaluated by a second-order interpolation method that is discussed in detail by Tomé et al. [25], and hence is not given here.

\subsection{Particle movement}

The last step in the calculation is to update the position of the moving free surface. The free surface is described by a set of marker particles and visualized by connecting these particles by straight lines (see Fig. 2). It can happen that two contiguous particles become too far apart or excessively close. In this case, an algorithm to insert or to delete particles is employed (for details, see [58]). The last step in the algorithm is to move the markers to their new positions by solving

$$
\frac{d \mathbf{x}_{P}}{d t}=\mathbf{v}^{(n+1)},
$$

for each particle $\mathbf{x}_{P}$ using the second-order modified Euler method, which gives,

$$
\mathbf{x}_{P}\left(t_{n+1}\right)=\mathbf{x}_{P}(t)+\frac{\delta t}{2}\left[\mathbf{v}\left(\mathbf{x}_{P}(t), t_{n+1}\right)+\overline{\mathbf{v}}\left(\overline{\mathbf{x}}_{P}\left(t_{n+1}\right), t_{n+1}\right)\right]
$$

where $\overline{\mathbf{x}}_{P}\left(t_{n+1}\right)=\mathbf{x}_{P}(t)+\delta t \mathbf{v}\left(\mathbf{x}_{P}(t), t_{n+1}\right)$. The velocities $\mathbf{v}\left(\mathbf{x}_{P}(t), t_{n+1}\right)$ and $\mathbf{\mathbf { v }}\left(\overline{\mathbf{x}}_{P}\left(t_{n+1}\right), t_{n+1}\right)$ are calculated using the updated velocity field $\mathbf{v}^{(n+1)}$ and are found from a bilinear interpolation involving the four nearest cell-velocities and the corresponding distances. Fig. 4 illustrates the velocities involved when computing the particle velocity $\mathbf{v}\left(\mathbf{x}_{P}(t), t_{n+1}\right)$.

\section{Verification and convergence results in simple flows}

To verify the accuracy of the methodology employed for calculating the integration nodes $t_{j}^{\prime}$ described in Section 3.2.1, predictions for shear and uniaxial elongational flows of a UCM fluid were compared to the corresponding analytic solutions, as follows.

Exact solution of shear flow of UCM fluids: In these flows, the velocity vector and the Finger tensor are given by

$$
\left\{\begin{array}{l}
u=\dot{\gamma} y, \\
v=0, \\
w=0,
\end{array} \quad \mathbf{B}_{t^{\prime}}(t)=\left(\begin{array}{ccc}
1+\dot{\gamma}^{2}\left(t-t^{\prime}\right)^{2} & \dot{\gamma}\left(t-t^{\prime}\right) & 0 \\
\dot{\gamma}\left(t-t^{\prime}\right) & 1 & 0 \\
0 & 0 & 1
\end{array}\right),\right.
$$

and the extra-stress components become

$$
\begin{aligned}
& \tau^{x x}(t)=a e^{\frac{-t}{\lambda}}\left(1+\dot{\gamma}^{2} t^{2}\right)+a\left(1-e^{\frac{-t}{\lambda}}\right)+a \dot{\gamma}^{2}\left[2 \lambda^{2}-e^{\frac{-t}{\lambda}}\left(t^{2}+2 \lambda t+2 \lambda^{2}\right)\right], \\
& \tau^{x y}(t)=a \dot{\gamma} \lambda\left(1-e^{\frac{-t}{\lambda}}\right), \tau^{x z}(t)=\tau^{y z}(t)=0, \\
& \tau^{y y}(t)=\tau^{z z}(t)=a .
\end{aligned}
$$

Exact solution for uniaxial elongational flow of UCM fluids: In this flow the velocity vector and Finger tensor components are given by

$$
\left\{\begin{array}{l}
u=\dot{\varepsilon} x, \\
v=-\frac{1}{2} \dot{\varepsilon} y, \\
w=-\frac{1}{2} \dot{\varepsilon} z .
\end{array} \quad \mathbf{B}_{t^{\prime}}(t)=\left(\begin{array}{ccc}
e^{2 \dot{\varepsilon}\left(t-t^{\prime}\right)} & 0 & 0 \\
0 & e^{-\dot{\varepsilon}\left(t-t^{\prime}\right)} & 0 \\
0 & 0 & e^{-\dot{\varepsilon}\left(t-t^{\prime}\right)}
\end{array}\right) .\right.
$$


Table 1

Relative errors obtained in the calculation of $\tau^{x x}$ for shear and elongational flows as a function of $N$.

\begin{tabular}{|c|c|c|c|c|}
\hline$N$ & Shear flow & & Uniaxial elongational flc & \\
\hline \multirow[t]{3}{*}{20} & Hulsen et al. & 0.000189 & Hulsen et al. & 0.00138 \\
\hline & Memory function & $6.668584 \times 10^{-7}$ & Memory function & 0.25664 \\
\hline & Geometric progression & $6.668584 \times 10^{-7}$ & Geometric progression & 0.09317 \\
\hline \multirow[t]{3}{*}{40} & Hulsen et al. & $1.56 \times 10^{-5}$ & Hulsen et al. & 0.00010 \\
\hline & Memory function & $6.668584 \times 10^{-7}$ & Memory function & 0.13545 \\
\hline & Geometric progression & $6.668584 \times 10^{-7}$ & Geometric progression & 0.00618 \\
\hline \multirow[t]{3}{*}{80} & Hulsen et al. & $1.29 \times 10^{-6}$ & Hulsen et al. & $8.2 \times 10^{-6}$ \\
\hline & Memory function & $6.668584 \times 10^{-7}$ & Memory function & 0.06971 \\
\hline & Geometric progression & $6.668584 \times 10^{-7}$ & Geometric progression & 0.00035 \\
\hline \multirow[t]{3}{*}{160} & Hulsen et al. & - & Hulsen et al. & - \\
\hline & Memory function & $6.668584 \times 10^{-7}$ & Memory function & 0.03462 \\
\hline & Geometric progression & $6.668584 \times 10^{-7}$ & Geometric progression & $2.14385 \times 10^{-5}$ \\
\hline \multirow[t]{3}{*}{320} & Hulsen et al. & - & Hulsen et al. & - \\
\hline & Memory function & $6.668584 \times 10^{-7}$ & Memory function & 0.01634 \\
\hline & Geometric progression & $6.668584 \times 10^{-7}$ & Geometric progression & $1.32904 \times 10^{-6}$ \\
\hline
\end{tabular}

It can be shown that the extra-stress components $\tau^{x x}$ and $\tau^{y y}$ are given by

$$
\begin{aligned}
& \tau^{x x}(t)=a e^{\left(\frac{-t(1-2 \lambda \dot{\varepsilon})}{\lambda}\right)}\left[1-\frac{1}{1-2 \lambda \dot{\varepsilon}}\right]+\frac{a}{1-2 \lambda \dot{\varepsilon}}, \\
& \tau^{y y}(t)=a e^{\left(-\frac{t(1+\lambda \dot{\varepsilon})}{\lambda}\right)}\left[1-\frac{1}{1+\lambda \dot{\varepsilon}}\right]+\frac{a}{1+\lambda \dot{\varepsilon}} .
\end{aligned}
$$

In equations (36)-(42), $\dot{\gamma}$ and $\dot{\varepsilon}$ denote constant shear and elongation rates while $\lambda$ and $a$ are the relaxation time of the fluid and the relaxation mode, respectively.

For negative values of $t^{\prime}$ the Finger tensor is set to that at $t^{\prime}=0$. The Finger tensor, for both shear and uniaxial flows, was numerically calculated by the explicit Euler method using the differential equations:

$$
\begin{aligned}
& \text { Shear flows Elongational flows } \\
& \left\{\begin{array} { l } 
{ \frac { \partial } { \partial t } B _ { t ^ { \prime } } ^ { y y } = 0 , } \\
{ \frac { \partial } { \partial t } B _ { t ^ { \prime } } ^ { x y } = \dot { \gamma } B _ { t ^ { \prime } } ^ { y y } , } \\
{ \frac { \partial } { \partial t } B _ { t ^ { \prime } } ^ { x x } = 2 \dot { \gamma } B _ { t ^ { \prime } } ^ { x y } . }
\end{array} \quad \left\{\begin{array}{l}
\frac{\partial}{\partial t} B_{t^{\prime}}^{x x}=2 \dot{\varepsilon} B_{t^{\prime}}^{x x}, \\
\frac{\partial}{\partial t} B_{t^{\prime}}^{y y}=-\dot{\varepsilon} B_{t^{\prime}}^{y y} .
\end{array}\right.\right.
\end{aligned}
$$

In elongational flows, the component $\tau^{x x}(t)$ exhibits a singularity at $\lambda \dot{\varepsilon}=\frac{1}{2}$ so that, to keep it bounded, the values used for $\lambda \dot{\varepsilon}$ are such that $\lambda \dot{\varepsilon}<\frac{1}{2}$.

To show the effects of the discretization of the interval $\left\{0, t_{n+1}\right\}$ using the geometric progression, the solution of the integrals to calculate the stress tensor in shear and elongational flows was numerically obtained. The following input data were used: $a=100 \mathrm{~Pa}, \lambda=1 \mathrm{~s}, t_{n+1}=10 \lambda, \dot{\gamma}=1 \mathrm{~s}^{-1}, \dot{\varepsilon}=0.3 \mathrm{~s}^{-1}, \delta t=10^{-6} \mathrm{~s}$ (used also to calculate the Finger tensor by equations (43)). The stress components were computed using both the inverse image of the memory function and the new scheme, the geometric progression technique to discretize the time interval $\left\{0, t_{n+1}\right\}$ and the analytic values of the stress were used to compute the corresponding relative errors $\left(\left|\tau_{\text {exact }}^{x x}-\tau_{\text {num }}^{x x}\right| /\left|\tau_{\text {exact }}^{x x}\right|\right)$. Table 1 lists those relative errors for $\tau^{x x}$ for both shear and uniaxial elongational flows for several values of $N$ and they are also plotted in Fig. 5. For comparison, the results obtained by the method of Hulsen et al. [60] for $N=20,40,80$ are also shown. It is seen in Fig. 5(a) that the relative errors for shear flows were the same for both the memory function and the geometric progression because the quadrature formula employed to solve the integrals is second order accurate and therefore, for this flow, it provides exact values. The error obtained, $6.668584 \times 10^{-7}$, is due to the convection of the Finger tensor by the explicit Euler method that is first order. With regard to the numerical solution for the uniaxial elongational flow, Fig. 5(b) shows that the errors obtained with the geometric progression are much smaller than those obtained using the inverse image of the memory function technique and gives similar results to those obtained by Hulsen et al. [60].

To better illustrate the effects of the distribution of the integration nodes $t_{j}^{\prime}$ in the computation of the stress tensor for uniaxial elongational flows, the component $\tau^{x x}$ was again calculated by both techniques, the memory function and the geometric progression for the discretization of the interval $\{0,10\}$, and $\tau^{x x}$ was calculated at $t_{n+1}=10 \mathrm{~s}$ using $N=30,50$ for $\dot{\varepsilon}$ in the range $\{0.1,0.49 \lambda\}$. The relative errors against the analytic solution are plotted in Fig. 6 , where it can be seen that for small values of $\dot{\varepsilon}$ both techniques produce similar relative errors. However, as $\dot{\varepsilon}$ increases and approaches 


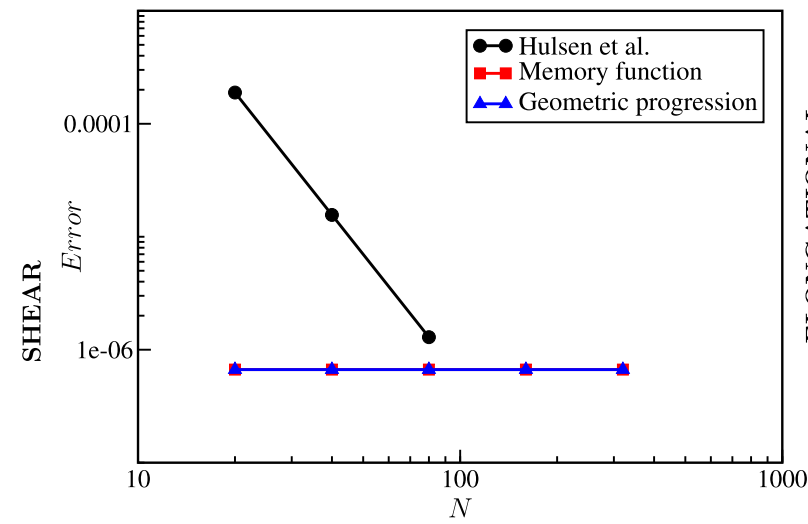

(a)

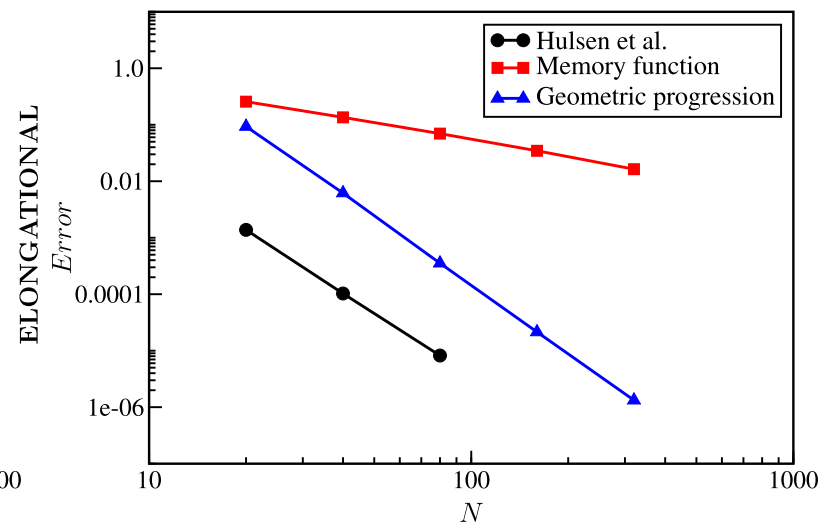

(b)

Fig. 5. Relative errors in the computation of $\tau^{x x}$ at $t=10 \lambda$ as a function of $N$ using $\dot{\gamma}=1 \mathrm{~s}^{-1}$ and $\dot{\varepsilon}=0.3 \mathrm{~s}^{-1}$ for shear (a) and elongational flows (b).

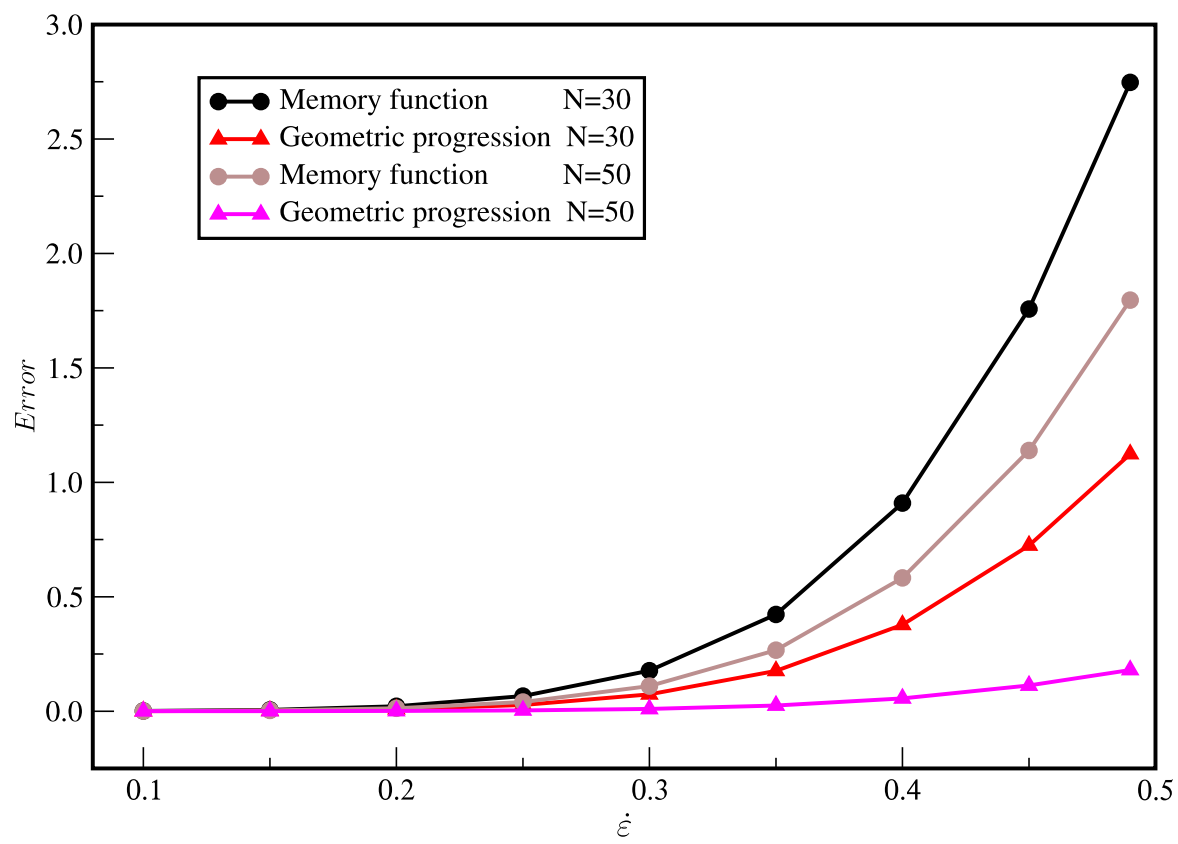

Fig. 6. Error in the calculation of $\tau^{x x}$ for $0.1 \leq \dot{\varepsilon} \leq 0.49, \lambda=1 \mathrm{~s}, a=100$ Pa using the memory function (circles) and the geometric progression (triangles) for $N=30,50$.

the singular value of 0.5 the errors obtained with the geometric progression are much smaller that those from the inverse image of the memory function. These results demonstrate that the geometric progression technique is superior to the inverse image of the memory function technique and therefore it will be adopted in this work to discretize the time range.

\section{Verification and convergence results for $\mathrm{K}-\mathrm{BKZ}$ fluids}

In this section, the numerical method described in this work was applied to simulate channel flow and a jet flowing onto a rigid plate of K-BKZ fluids on several grids. The verification of the numerical technique is provided by comparing to predictions of channel flow with the analytic solution for a 1-mode K-BKZ fluid and the mesh independence is assessed by mesh refinement.

\subsection{Mesh independence in channel flow}

The numerical method described in Section 3 was applied to simulate the flow of a K-BKZ fluid in a channel of length $10 L$ and height $L$, where $L=1 \mathrm{~cm}$. At the channel entrance, a nondimensional parabolic velocity profile given by

$$
u(y / L)=-4(y / L-0.5)^{2}+1
$$


Table 2

Definition of the fluid used in the simulation of channel flow. Data taken from Quinzani et al. [63].

\begin{tabular}{|c|c|c|c|}
\hline \multicolumn{4}{|c|}{ FLUID S1 } \\
\hline \multicolumn{4}{|c|}{$\rho_{0}=801.5 \mathrm{~kg} / \mathrm{m}^{3}, \alpha=10, \beta=0.7, \lambda_{\text {ref }}=0.06 \mathrm{~s}, \eta_{0}=1.424$ Pa.s } \\
\hline$k$ & $\lambda_{k}[\mathrm{~s}]$ & $a_{k}[\mathrm{~Pa}]$ & $\eta_{k}[$ Pa.s $]$ \\
\hline 1 & 0.6855 & 0.058352 & 0.0400 \\
\hline 2 & 0.1396 & 1.664756 & 0.2324 \\
\hline 3 & 0.0389 & 14.560411 & 0.5664 \\
\hline 4 & 0.0059 & 99.152542 & 0.5850 \\
\hline
\end{tabular}

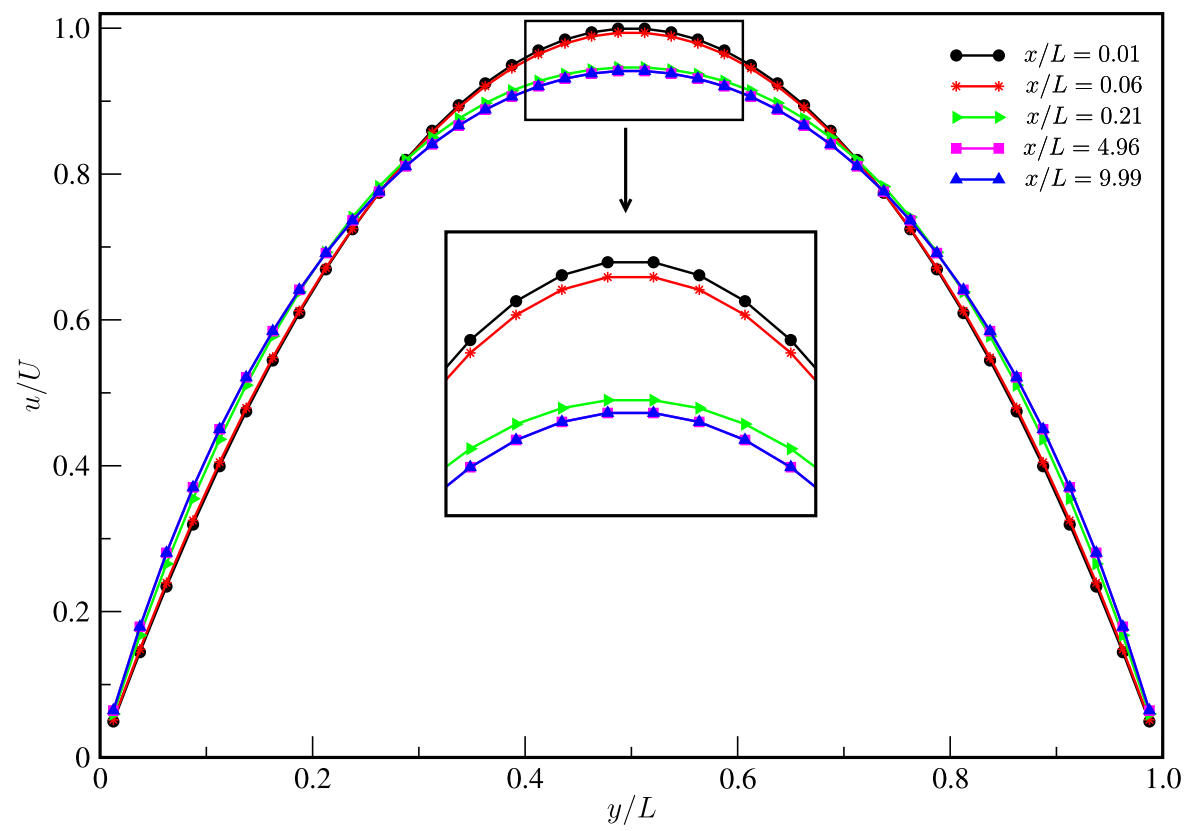

Fig. 7. $u$-Velocity profiles obtained in the simulation of the channel flow at $t=100 \mathrm{~s}$ through the channel. Results shown at several values of $x / L$ : 0.01 (black), 0.06 (red), 0.21 (green), 4.96 (violet (overwritten by the blue curve)), 9.99 (blue). (For interpretation of the references to color in this figure legend, the reader is referred to the web version of this article.)

was employed. The scaling parameters were the centreline velocity, $U=0.167 \mathrm{~m} \mathrm{~s}^{-1}$ and the channel width $L$; the tolerance for the Poisson equation solver was EPS $=10^{-10}$. The fluid simulated was FLUID S1 which is defined in Table 2. In this flow we had $R e=0.93, W i=1$ and the number of deformation fields was $N=100$. To verify mesh convergence of the results, the flow was simulated using the meshes I $(100 \times 10)$, II $(200 \times 20)$ and III $(400 \times 40)$ until $t=100 \mathrm{~s}$. It is expected that after $t=100 \mathrm{~s}$ from start-up, steady state has been reached and this is confirmed by the $u$-profiles displayed in Fig. 7 where we can see that the velocity in the channel quickly develops to the K-BKZ solution that is calculated at the end of the channel.

The computed transverse profile of the velocity and the extra-stress tensor are plotted in Fig. 8 and for the velocity plot a zoom in of the profiles in the vicinity of the centreline is displayed. It is clear that the fully developed K-BKZ profiles are not parabolic (for comparison, the Newtonian profile is shown in Fig. 8(a)).

\subsection{Verification in channel flow for a 1-mode K-BKZ fluid}

The authors have developed an analytic solution of a 1-mode K-BKZ model for fully developed channel flows which was employed to verify the numerical technique developed in this work. The construction of this analytic solution is detailed in Appendix A.

The channel flow was simulated using a 1 -mode K-BKZ fluid with $\lambda_{1}=\lambda_{\text {ref }}=0.1396 \mathrm{~s}, \quad a_{1}=1.6648 \mathrm{~Pa}, \rho_{0}=$ $801.5 \mathrm{~kg} \mathrm{~m}^{-3}$ and $\eta_{0}=\lambda_{1} * a_{1}=0.2324 \mathrm{Pas}$ for a flow with a centreline velocity $U=0.025 \mathrm{~m} \mathrm{~s}^{-1}$ and $L=1 \mathrm{~cm}$ which gave $R e=\frac{\rho_{0} U L}{\eta_{0}}=0.8621$ and $W i=\lambda_{\text {ref }} \frac{U}{L}=0.3490$. This flow was simulated on meshes $I(100 \times 10), I I(200 \times 20)$, $I I I(300 \times 30)$ and $I V(400 \times 40)$ from start-up until $t=100 \mathrm{~s}$. The results obtained for $\partial u(y) / \partial y$ are plotted in Fig. 9 while the results for $\tau^{x x}$ and $\tau^{y y}$ are displayed in Fig. 10, where it can be seen that the agreement between the numerical and the analytic solutions is good. It is observed that the numerical solutions converge to the analytic solution when the mesh is refined. 


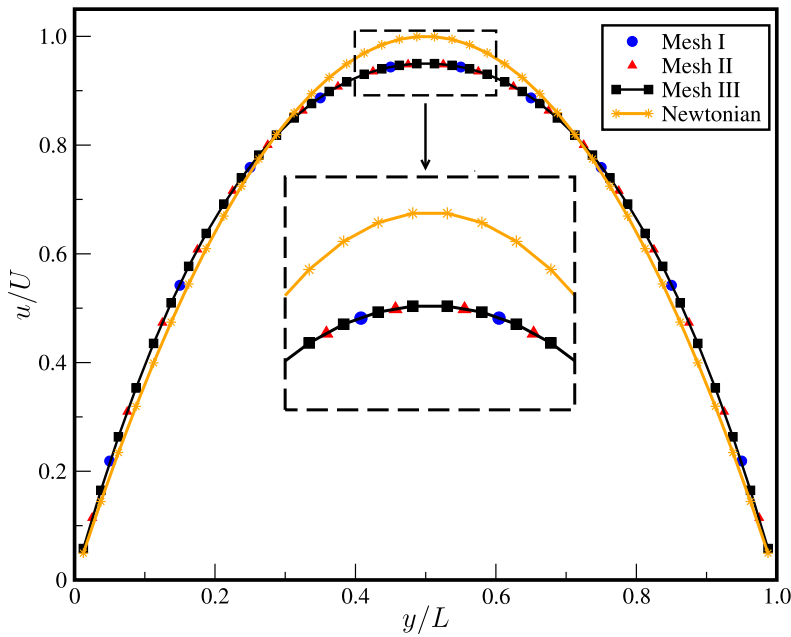

(a)

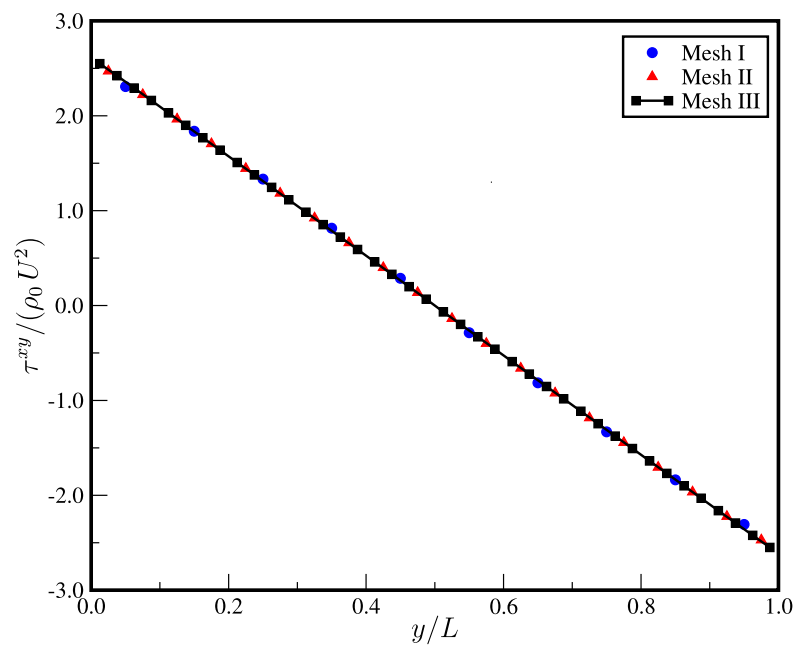

(c)

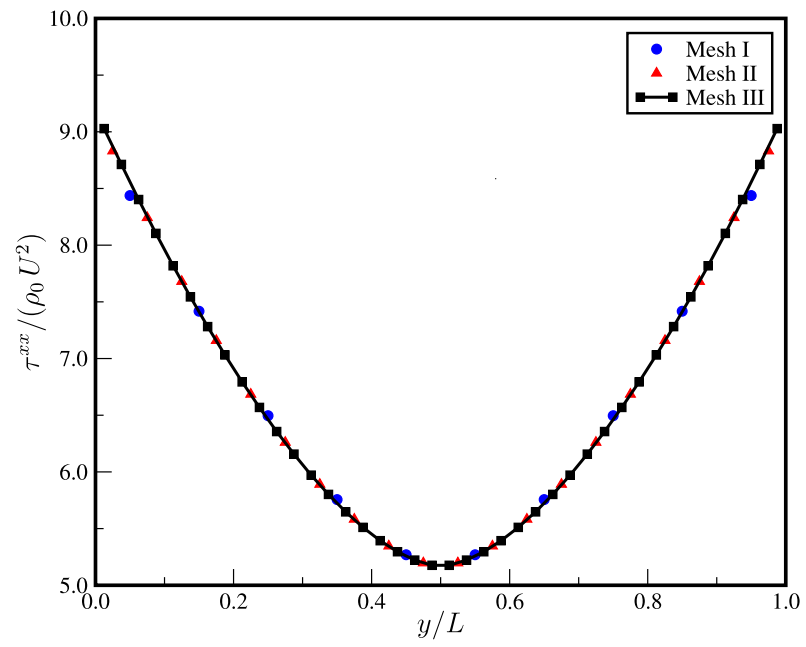

(b)

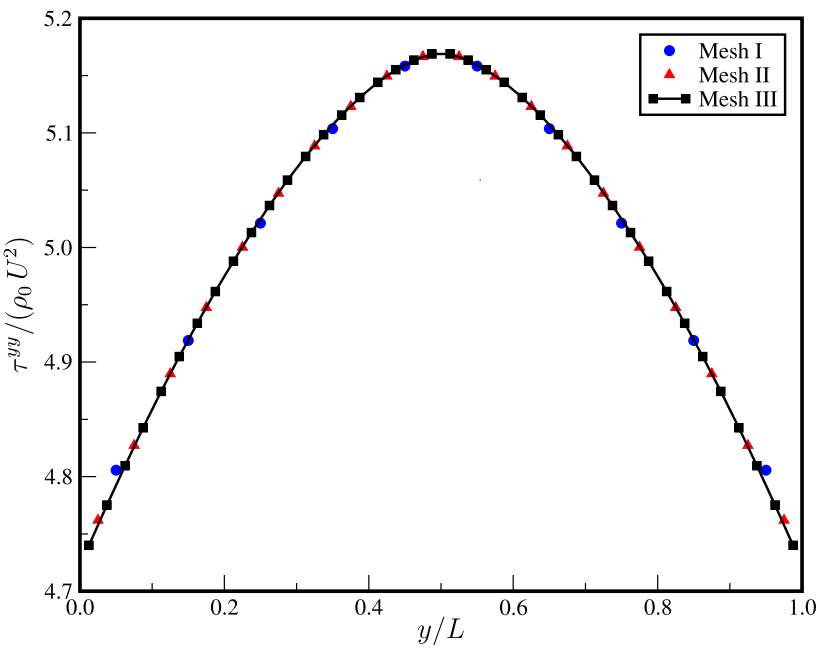

(d)

Fig. 8. Numerical solutions of fully developed channel flow of FLUID S1 using meshes I, II and III. (a) $u(y) / U$; (b) $\tau^{x x}(y) /\left(\rho_{0} U^{2}\right)$; (c) $\tau^{x y}(y) /\left(\rho_{0} U^{2}\right)$; (d) $\tau^{y y}(y) /\left(\rho_{0} U^{2}\right)$. The yellow curve in (a) represents the Newtonian velocity profile. (For interpretation of the references to color in this figure legend, the reader is referred to the web version of this article.)

In addition, to quantify the convergence of the numerical method, the errors obtained on meshes $M_{l}$ (where $M_{l}$ denotes either I, II, III or IV) were computed by the grid function norm given by

$$
E\left(\bullet, M_{l}\right)=\sqrt{H_{l} \sum_{j=1}^{J_{l}}\left[\operatorname{Sol}_{\text {an }}\left(\bullet, M_{l}\right)-\operatorname{Sol}_{\text {num }}\left(\bullet, M_{l}\right)\right]^{2}},
$$

where $J_{l}$ denotes the value of $j_{\max }$ and $H_{l}$ is the spacing of mesh $M_{l}$. These errors were computed with equation (45) using the values on the transversal section of the channel before the channel exit and are displayed in Table 3 . It is seen that the computed errors diminish as the mesh is refined, decaying to less than $1 \%$ in the finest mesh employed. Furthermore, the pressure gradient was calculated (see the last column of Table 3) which also displays convergence to the corresponding analytic value.

The calculated order of convergence between the meshes employed is given in Table 4 which shows values between 1.6 and 1.8. These values are not exactly equal 2 , but are close to the expected order of 2 . These differences may be justified by the fact that the computation of the errors involved points lying in the vicinity of the boundaries where the equations were solved by first order approximations. Moreover, to compute the values of the analytic solution, on the required grid points, the Simpson $1 / 3$ quadrature formula and linear interpolation were employed. This may have affected the accuracy of the numerical solutions justifying the values found between 1.6 and 1.8 . 


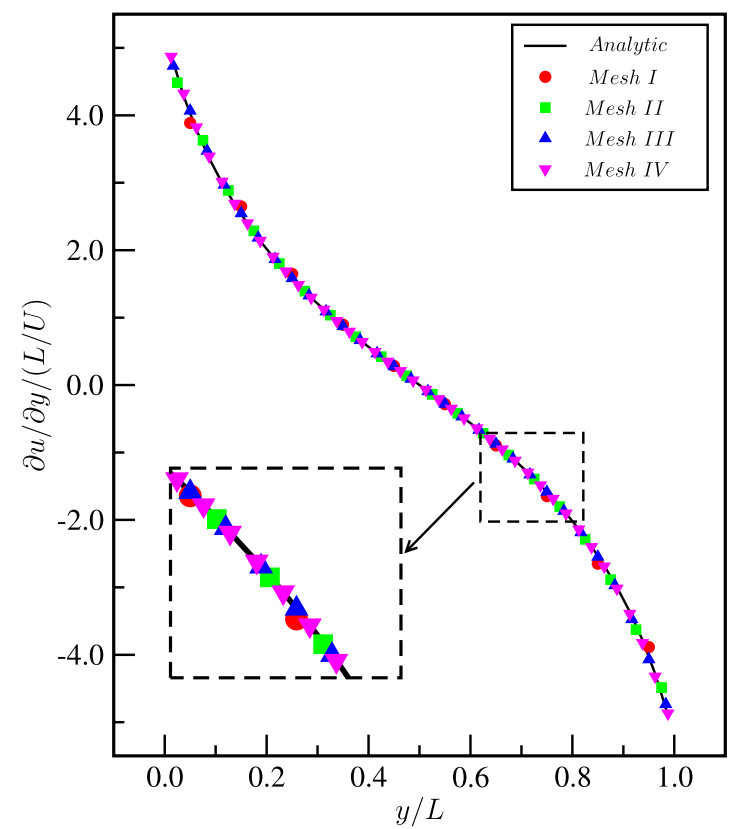

Fig. 9. Numerical solution for the fully-developed channel flow of a 1-mode K-BKZ fluid with mesh refinement. Comparison of $\frac{U}{L} \partial u(y) / \partial y$ obtained on meshes I, II, III, IV, with the analytic solution.
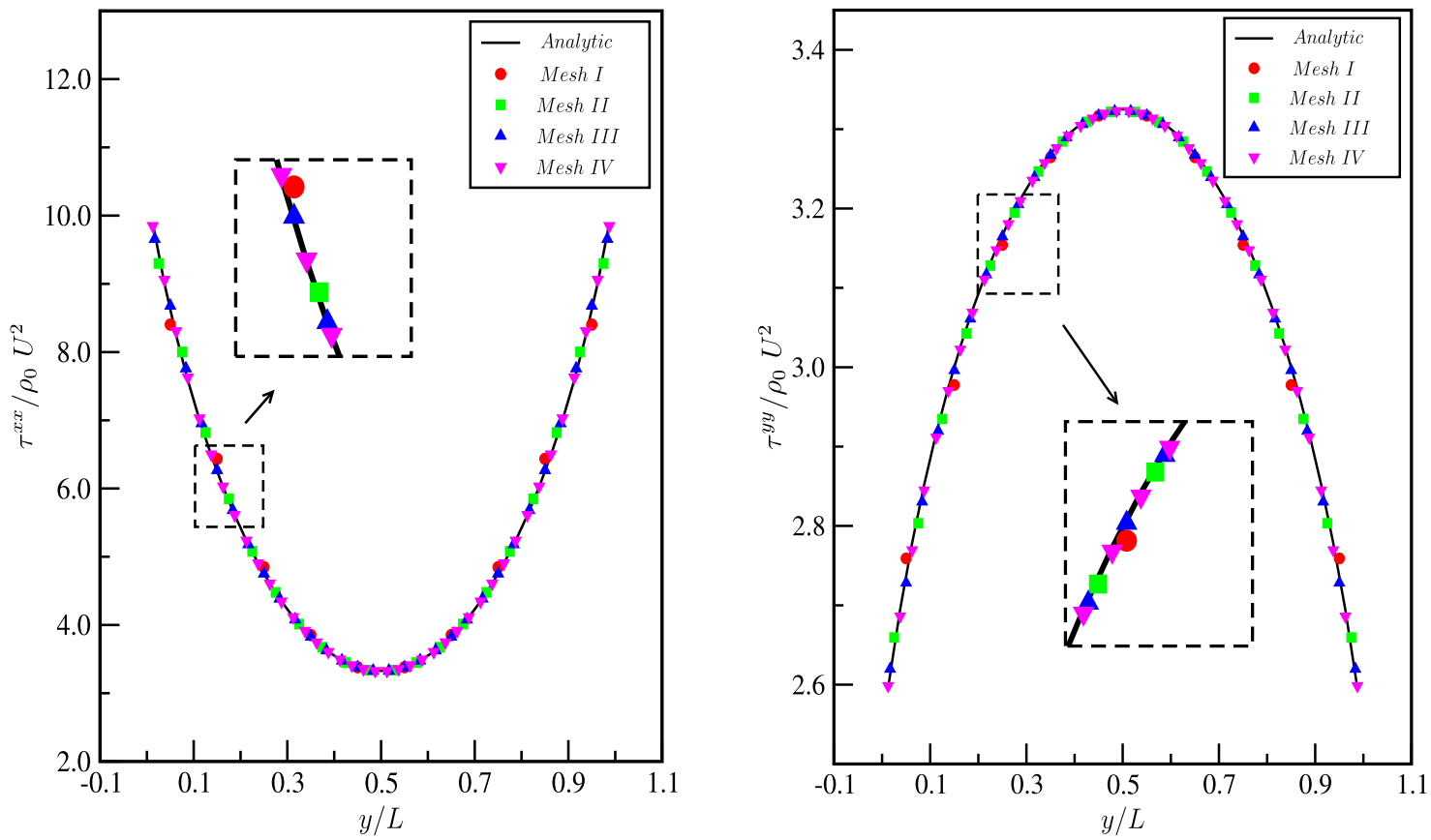

Fig. 10. Numerical solutions of 1-mode K-BKZ fully developed channel flow with mesh refinement. Comparison of $\tau^{x x}(y) /\left(\rho_{0} U^{2}\right)$ and $\tau^{y y}(y) /\left(\rho_{0} U^{2}\right)$ obtained on meshes $I, I I, I I I, I V$, with the analytic solution.

\subsection{Verification of the method on moving free surface flows}

The methodology presented in this paper has been verified on two free surface problems:

i) Time-dependent flow of a jet flowing down onto a rigid plate;

ii) Simulation of the extrudate swell of FLUID M1 and comparison with the results of Mitsoulis [44].

The results obtained are presented next. 
Table 3

Relative errors of $\frac{\partial u(y)}{\partial y}, \tau^{x x}, \tau^{y y}$, obtained in the calculation of channel flow of a 1-mode K-BKZ fluid on meshes I, II, III, IV using equation (45). Last column displays the average value of $p_{x} \approx$ $\left(\sum_{j=1}^{J_{l}} \frac{p_{i_{\text {out }}, j}-p_{i_{\text {out }-1}, j}}{\delta x}\right) / J_{l}$, where $i_{\text {out }}$ denotes the $i$-index of the column before the channel exit.

\begin{tabular}{lllll}
\hline Meshes $M_{l}$ & $\frac{\partial u(y)}{\partial y}$ & $\tau^{x x}(y)$ & $\tau^{y y}(y)$ & $\begin{array}{l}\text { Exact value } \\
p_{x}=-6.42226\end{array}$ \\
\hline$I$ & & & & -6.32040 \\
$I I$ & $0.2694407 \mathrm{E}-01$ & $0.4184212 \mathrm{E}-01$ & $0.480304 \mathrm{E}-02$ & -6.37054 \\
$I I I$ & $0.871597 \mathrm{E}-02$ & $0.1317354 \mathrm{E}-01$ & $0.162794 \mathrm{E}-02$ & -6.39534 \\
$I V$ & $0.421417 \mathrm{E}-02$ & $0.619823 \mathrm{E}-02$ & $0.88220 \mathrm{E}-03$ & -6.40965 \\
\hline
\end{tabular}

Table 4

Order of convergence obtained on meshes I, II, III, IV using the results presented in Table 3.

\begin{tabular}{llll}
\hline Meshes $M_{l}$ & $\frac{\partial u(y)}{\partial y}$ & $\tau^{x x}(y)$ & $\tau^{y y}(y)$ \\
\hline$I$ & - & - & - \\
$I I$ & 1.628 & 1.667 & 1.561 \\
$I I I$ & 1.792 & 1.859 & 1.511 \\
$I V$ & 1.624 & 1.718 & 1.164 \\
\hline
\end{tabular}

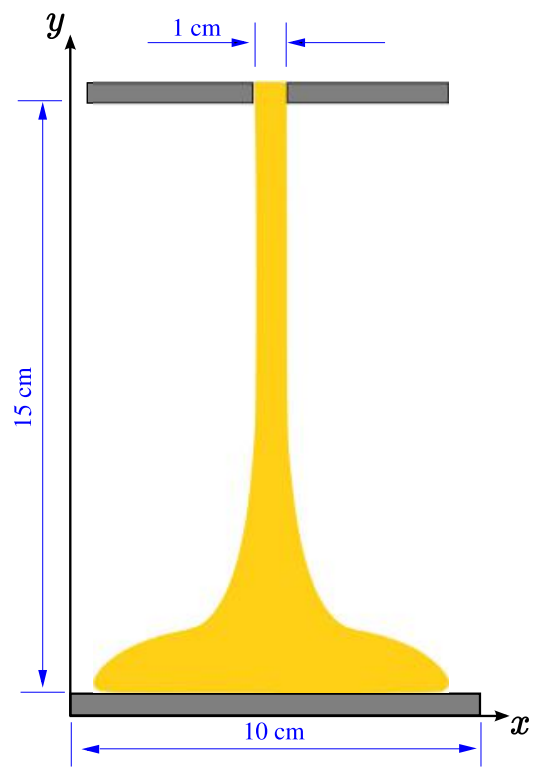

Fig. 11. Description of the domain used in the simulation of a jet impinging on a rigid plate.

Table 5

Data used in the simulation of a jet impinging on a rigid plate.

\begin{tabular}{lllllll}
\hline$D$ & $U$ & $H$ & $H / D$ & $R e$ & $W i$ & $F r$ \\
\hline $1 \mathrm{~cm}$ & $0.5 \mathrm{~m} \mathrm{~s}^{-1}$ & $15 \mathrm{~cm}$ & 15 & 0.45 & 7 & 1.59 \\
\hline
\end{tabular}

\subsubsection{Numerical simulation of a jet impinging on a rigid plate}

To show convergence of the numerical method in simulating time-dependent free surface flows, a jet of diameter $D=1 \mathrm{~cm}$ of a K-BKZ fluid impinging on a rigid surface was simulated and mesh refinement studies were performed. A computational domain of size $10 \mathrm{~cm} \times 15 \mathrm{~cm}$ was employed, as illustrated in Fig. 11, and the meshes used in these simulations were M1: $40 \times 60(\delta x=D / 4)$; M2: $80 \times 120(\delta x=D / 8)$; M3: $100 \times 150(\delta x=D / 10)$; M4: $200 \times 300(\delta x=D / 20)$; M5: $300 \times 450(\delta x=D / 30)$ and M6: $400 \times 600(\delta x=D / 40)$. The remaining data used are listed in Table 5. The fluid employed was FLUID $B$ whose properties are displayed in Table 6 (data taken from [44]). In this study, the inlet conditions were: $u(x)=0, v(x)=U, \tau^{x x}(x)=\tau^{x y}(x)=\tau^{y y}(x)=0$. 
Table 6

K-BKZ fluid model parameters used in the jet impinging flow case. Data defining FLUID B (from Mitsoulis [44]).

FLUID B

$\rho_{0}=880 \mathrm{~kg} / \mathrm{m}^{3}, \alpha=25286, \beta=0.1, \lambda_{\text {ref }}=0.14 \mathrm{~s}, \eta_{0}=9.79$ Pa s

\begin{tabular}{llll}
\hline$k$ & $\lambda_{k}$ & $a_{k}$ & $\eta_{k}=\lambda_{k} * a_{k}$ \\
\hline 1 & $1.12 \times 10^{-4} \mathrm{~s}$ & $6.67 \times 10^{4} \mathrm{~Pa}$ & $7.470 \mathrm{~Pa} . \mathrm{s}$ \\
2 & $4.12 \times 10^{-2} \mathrm{~s}$ & $1.83 \times 10^{1} \mathrm{~Pa}$ & 0.754 Pa.s \\
3 & $8.64 \times 10^{-1} \mathrm{~s}$ & $1.83 \times 10^{0} \mathrm{~Pa}$ & $1.581 \mathrm{~Pa} . \mathrm{s}$ \\
\hline
\end{tabular}

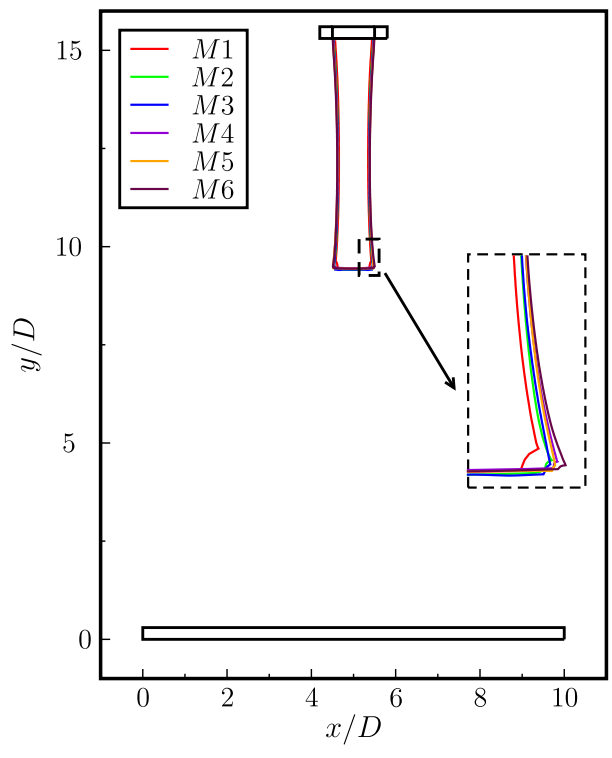

$t=0.10 \mathrm{~s}$

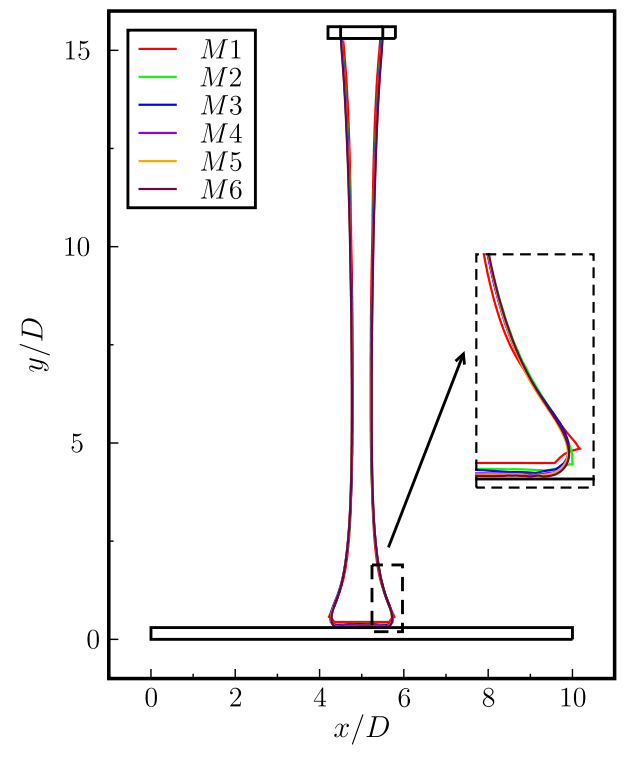

$t=0.20 \mathrm{~s}$

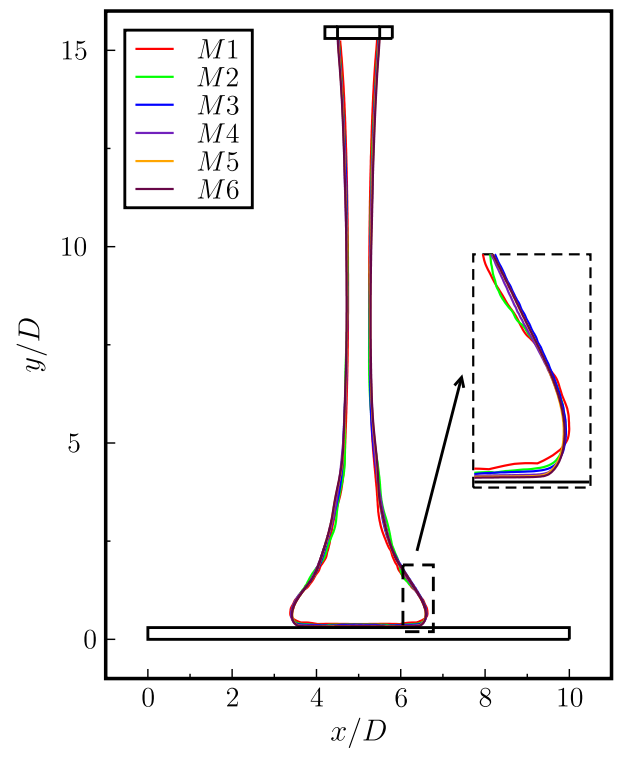

$t=0.30 \mathrm{~s}$

Fig. 12. Simulation of a jet impinging onto a rigid surface. Fluid flow visualization at selected times on meshes M1, M2, M3, M4, M5 and M6.

The results obtained are displayed in Fig. 12, which shows several snapshots taken at selected times during the calculations. Analyzing Fig. 12, one can see that the surface profiles obtained on the coarse meshes approximate the surface profile from mesh M6. In particular, the surface profiles of meshes M4, M5 and M6 are very near each other and from the 
Table 7

Data defining FLUID M1 (from [44]).

\begin{tabular}{lll}
\hline \multicolumn{2}{l}{ FLUID M1 } \\
\hline$\rho_{0}=868 \mathrm{~kg} / \mathrm{m}^{3}, \eta_{0}=2.4 \mathrm{Pas}$ \\
$\alpha=34214, \beta=0.1, \lambda_{\text {ref }}=0.081 \mathrm{~s}$ \\
\hline$k$ & $\lambda_{k}$ & $a_{k}$ \\
\hline 1 & $4.887 \times 10^{-4} \mathrm{~s}$ & $3129.5 \mathrm{~Pa}$ \\
2 & $4.464 \times 10^{-2} \mathrm{~s}$ & $5.0917 \mathrm{~Pa}$ \\
3 & $2.8384 \times 10^{-1} \mathrm{~s}$ & $2.2783 \mathrm{~Pa}$ \\
\hline
\end{tabular}

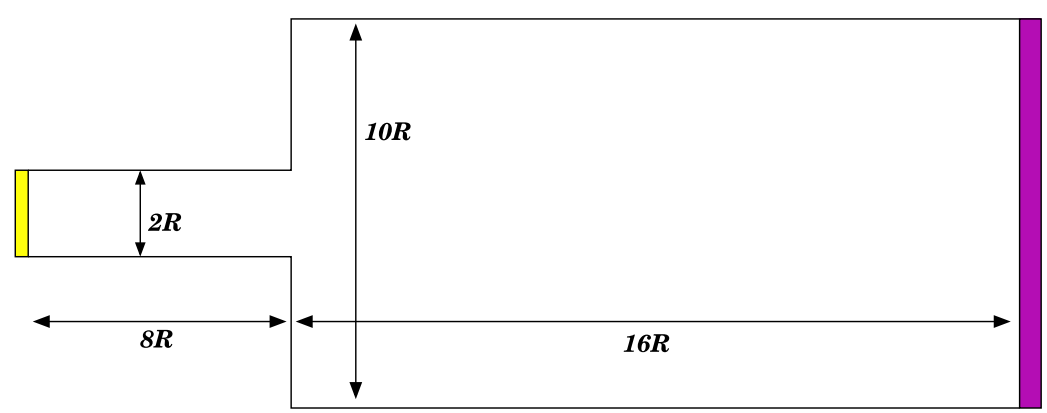

Fig. 13. Description of the computational domain. (For interpretation of the references to color in this figure, the reader is referred to the web version of this article.)

Table 8

Data employed in the simulation of the extrudate swell.

\begin{tabular}{ll}
\hline$R e=1.3 \quad W i=0.32$ & $g_{x}=g_{y}=0$ \\
\hline Mesh employed: & \\
$80 \times 192\left(\delta_{x} / L=\delta_{y} / L=0.0625\right)$ & \\
\hline
\end{tabular}

results, it is difficult to distinguish each one. In our opinion, this demonstrates the convergence of the free surface with mesh refinement.

\subsubsection{Simulation of the extrudate swell of FLUID M1}

We performed a simulation of the extrudate swell of FLUID M1 [44] and compared the results obtained with those presented by Mitsoulis [44]. The data defining FLUID M1 are reproduced in Table 7.

In his paper, Mitsoulis carried out a number of simulations of the extrudate swell using this FLUID M1 by varying the values of the apparent shear rate, $\dot{\gamma}_{a}$, which is defined in terms of the volumetric flow rate $Q$ and the width $L$ of the channel, by (see [44]):

$$
\dot{\gamma}_{a}=\frac{3 U}{L}
$$

where $U$ is a characteristic velocity. The viscoelasticity of the fluid was measured in terms of the stress ratio, $S_{R}$, given by [64]

$$
S_{R}=\frac{N_{1, w}}{2 \tau_{, w}^{x y}}=\frac{\left(\tau^{x x}-\tau^{y y}\right)_{, w}}{2 \tau_{, w}^{x y}},
$$

where $N_{1, w}$ and $\tau_{, w}^{x y}$ are evaluated using the calculated stresses at the die wall. Results were reported for values of $\dot{\gamma}_{a}=$ $9,12,15,18,21\left(\mathrm{~s}^{-1}\right)$. For each value of $\dot{\gamma}_{a}$, the calculated extrudate ratio $\chi$ and $S_{R}$ were presented.

To simulate this problem, the flow domain used is depicted in Fig. 13 in which the yellow and pink regions denote inflow and outflow boundaries, respectively.

At the fluid entrance (inflow), the velocity was given by a parabolic profile in equation (44) and the Finger tensor components were defined as in equation (36) while at the outflow, both the velocity and the Finger tensor obeyed homogeneous Neumann conditions.

To verify our numerical method on this application, we used $L=2 R=3 \mathrm{~cm} ; U=12 \mathrm{~cm} / \mathrm{s}$ and considered the case of $\dot{\gamma}=12 \mathrm{~s}^{-1},(U / L=4)$ and performed a calculation until steady state flow was achieved. In this simulation we used the input data displayed in Table 8. 
(a) $\mathrm{t}=0.5 \mathrm{~s}$

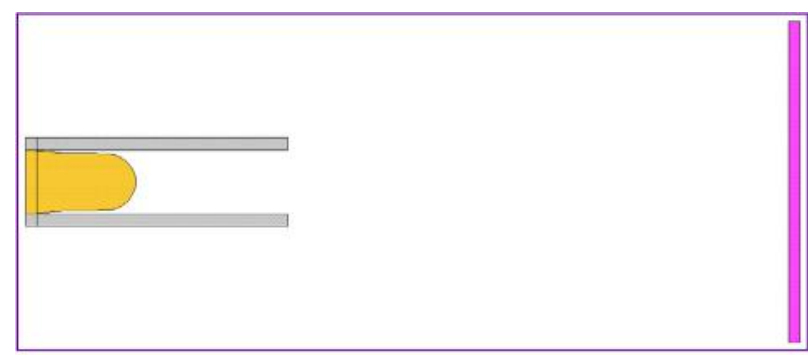

(c) $\mathrm{t}=2.75 \mathrm{~s}$

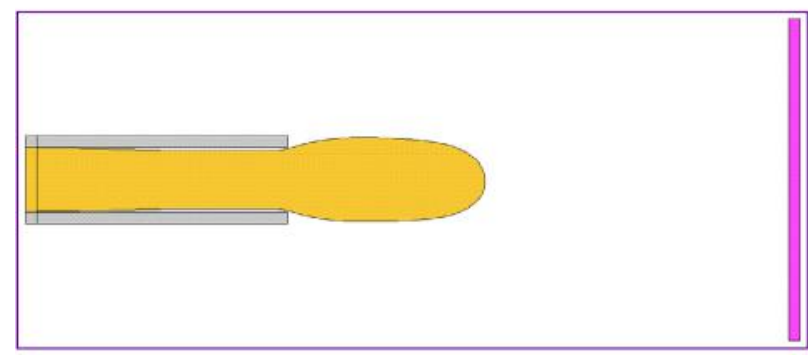

(e) $\mathrm{t}=8.0 \mathrm{~s}$

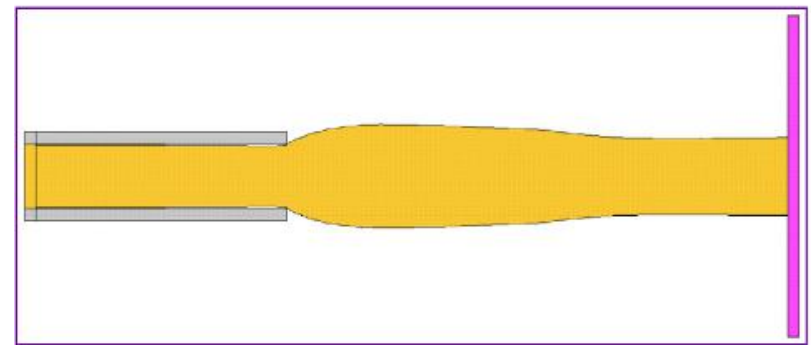

(b) $\mathrm{t}=1.25 \mathrm{~s}$

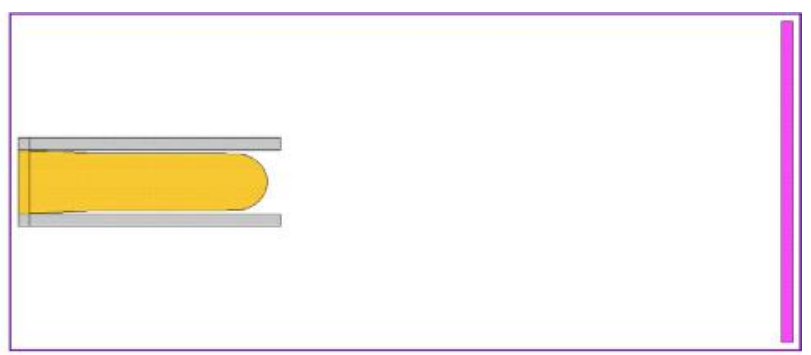

(d) $\mathrm{t}=4.5 \mathrm{~s}$

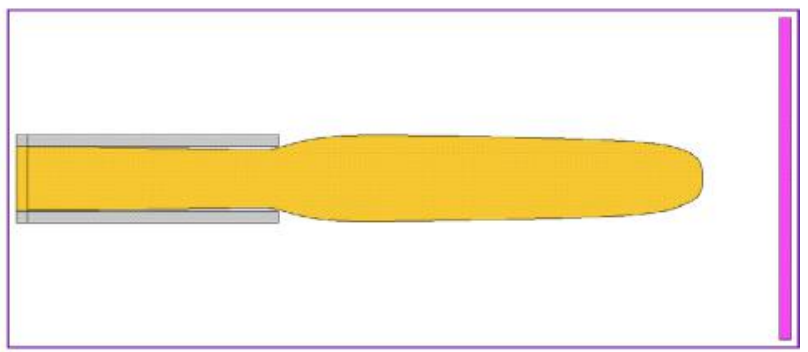

(f) $\mathrm{t}=32.5 \mathrm{~s}$

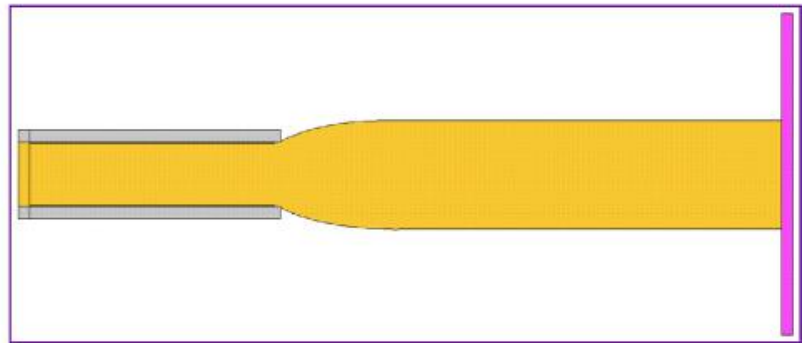

Fig. 14. Numerical simulation of the extrudate swell: fluid flow visualization at different times.

The simulation started with an empty domain and the fluid was injected through the channel entrance until it flowed out through the channel exit and extruded into the air where the stresses were relaxed, making the fluid swell. Fig. 14 displays several snapshots of the fluid configuration at different times. By time $t=32.5 \mathrm{~s}$ (Fig. 14(f)), we expect that a steady state has been established.

A comparison with the free surface profile obtained by Mitsoulis [44] is displayed in Fig. 15, where it can be seen that the agreement between the two solutions is good. The values of the extrudate ratio $(\chi)$ and the stress ratio $\left(S_{R}\right)$ obtained in this simulation, together with the values found by Mitsoulis [44], are displayed in Table 9. We can see in Table 9 that the agreement between the two solutions is very good. This verifies the numerical method developed in this work on complex free surface problems.

\section{Numerical simulation of jet buckling of K-BKZ fluids}

The flow produced by a jet flowing onto a rigid plate has application in many industrial processes as for example container filling. This problem has been investigated by many researchers (e.g. [65-67]) and it is known that, after jet impingement, various phenomena can be observed, such as, for instance, the phenomenon known as jet buckling. This is a physical instability in which, due to viscous forces and compression waves, the jet is unable to flow radially and accumulates onto itself causing the occurrence of jet buckling (see Fig. 16(a)). This problem was experimentally and theoretically studied by Cruickshank and Munson [65] for Newtonian fluids, who derived conditions based on the jet width ( $D$ ), height of the nozzle from which the jet is issued $(H)$ and Reynolds number $(R e)$, for predicting 'jet buckling'. In their analyses, Cruickshank and Munson [65] found that a two-dimensional jet will undergo 'jet buckling' if conditions below are satisfied

$$
\operatorname{Re}<0.56 \text { and } H / D>10 \text {. }
$$

These conditions have been verified by many researchers who simulated this phenomenon in two and three dimensions employing Newtonian and non-Newtonian fluids governed by differential models (see $[7,9,13,61,68,69]$ ). Notwithstanding, 


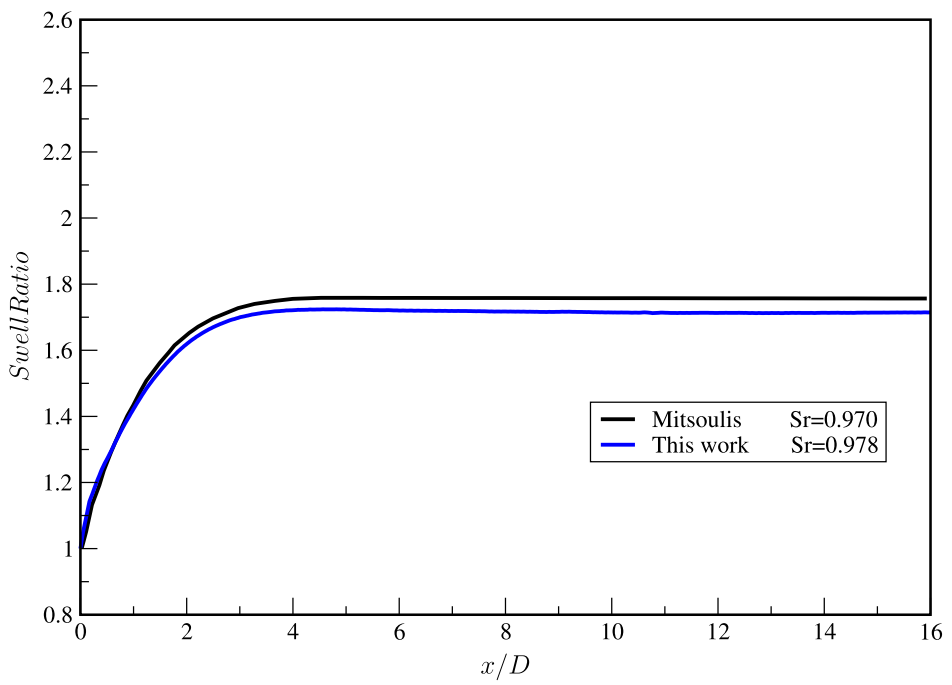

Fig. 15. Numerical simulation of extrudate swell. Comparison with the results of Mitsoulis [44]. $R e=1.3, W i=0.32$.

\section{Table 9}

Values of $\chi$ and $S_{R}$ obtained in the simulations and comparison with the results of Mitsoulis [44].

\begin{tabular}{lll}
\hline & $\chi$ & $S_{R}$ \\
\hline This work & $\mathbf{1 . 7 2}$ & $\mathbf{0 . 9 7 8}$ \\
Mitsoulis [44] & $\mathbf{1 . 7 6}$ & $\mathbf{0 . 9 7}$ \\
\hline
\end{tabular}

(a)

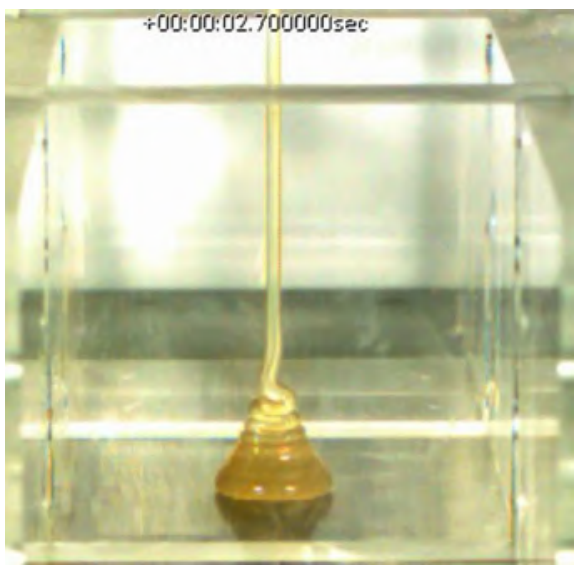

(b)

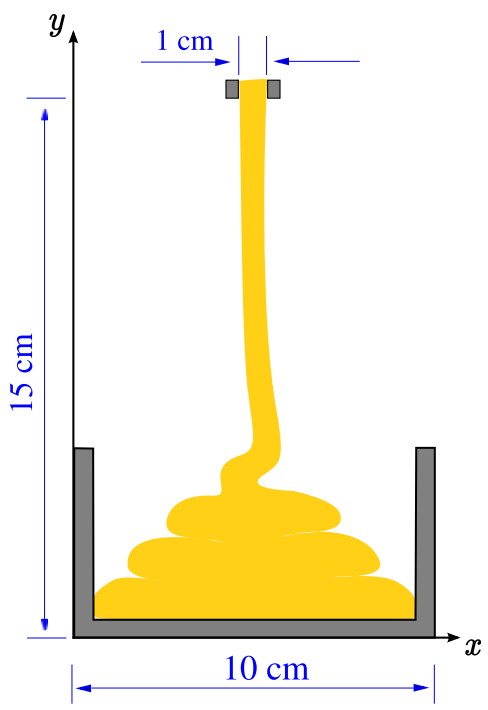

Fig. 16. (a) An example of jet buckling of a Newtonian fluid (from Tomé at al. [70]); (b) description of the domain used in the simulation of jet impingement.

the application of the integral K-BKZ constitutive equation to flows possessing moving free surfaces, as it happens in the problem of 'jet buckling', using the existing techniques has not yet been demonstrated.

In this section, it is demonstrated that the integral K-BKZ model can be employed to simulate time-dependent moving free surface flows. To this purpose, the numerical method presented in Section 3 was applied to simulate 'jet buckling' of fluids modeled by the K-BKZ integral constitutive equation. The fluid known as FLUID B, as it is described in Table 6, was employed in the simulations. A computational domain of $10 \mathrm{~cm} \times 15 \mathrm{~cm}$ was considered and a mesh with grid spacing $\delta x=\delta y=1 \mathrm{~mm}$ was used. A nozzle of width $D=1 \mathrm{~cm}$ was positioned at a height $H=15 \mathrm{~cm}$ above the bottom of an empty rectangular open box of dimensions $10 \mathrm{~cm} \times 5 \mathrm{~cm}$ (as illustrated in Fig. 16(b)), from which a jet was issued at various velocities. Gravity was acting downwards with $g_{y}=-9.81 \mathrm{~m} \mathrm{~s}^{-2}$. 
Table 10

Data employed in the simulation of an impinging jet of FLUID $B$.

\begin{tabular}{llll}
\hline$D=1 \mathrm{~cm}, H=15 \mathrm{~cm}, H / D=15, N=100$ & & \\
\hline$U\left(\mathrm{~m} \mathrm{~s}^{-1}\right)$ & $R e$ & $W i$ & $F r$ \\
\hline 0.2 & 0.17 & 2.8 & 0.60 \\
0.4 & 0.35 & 5.6 & 1.27 \\
0.6 & 0.53 & 8.4 & 1.91 \\
\hline
\end{tabular}
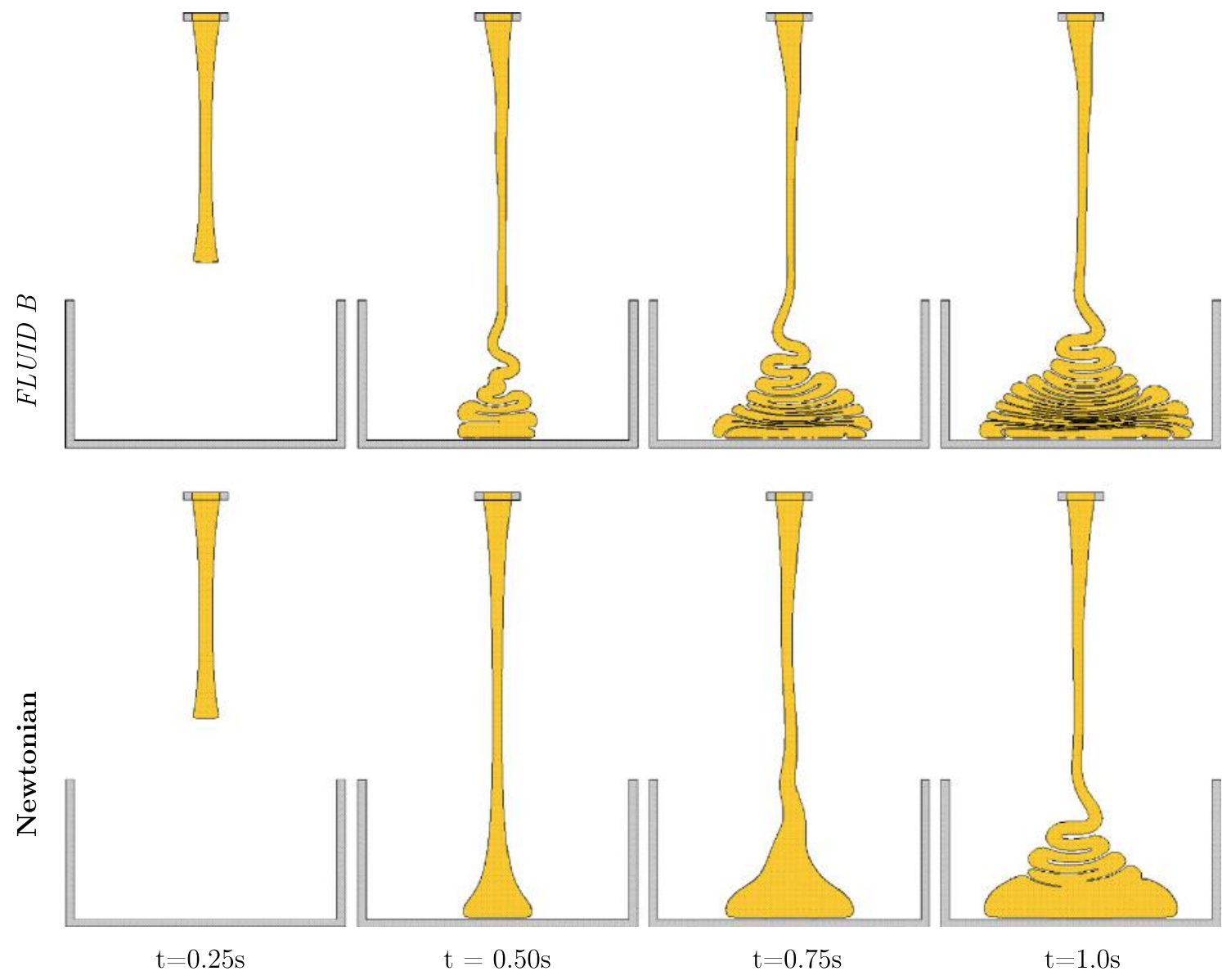

Fig. 17. Numerical simulation of impinging Newtonian and FLUID B jets with $U=0.2 \mathrm{~m} \mathrm{~s}^{-1}$. Fluid flow visualization at selected times.

The values of the velocity $U$ used and the nondimensional numbers, $R e, W i, F r$, involved in these simulations are displayed in Table 10 where it can be seen that, for the velocities $U=0.2,0.4,0.6 \mathrm{~m} \mathrm{~s}^{-1}$, the Cruickshank conditions (48) are satisfied and therefore, it is expected that the jet with Newtonian fluid undergoes buckling. With regard to the jet containing FLUID B, a similar analysis about the phenomenon of jet buckling has not yet been made. In these simulations we used a constant inlet velocity and the components of the stress tensor were set to zero. Naturally, in a real situation the flow and swell at the exit of the die will be different due to the flow pre-history, which can be minimized by a sufficiently long flow inlet channel, and the adaptation of the flow to the downstream outlet flow typical in low Reynolds number flows. However, we preferred not to show this effect on these simulations as the interest is focused on what happens after the jet flowed onto the bottom of the container.

The results of these simulations are shown in Figs. 17, 18 and 19 in which it can be seen that for the velocities $U=0.2,0.4 \mathrm{~ms}^{-1}$ (Figs. 17 and 18), both Newtonian and K-BKZ jets present jet buckling. However, for the velocity $U=0.6 \mathrm{~m} \mathrm{~s}^{-1}$, Fig. 19 shows that only the jet with FLUID $B$ undergoes buckling which we believe is due to the elasticity of the fluid. The analysis of Cruickshank and Munson was based on experiments and therefore it may not be sufficiently accurate which may justify why the Newtonian jet with $R e=0.53$ did not present buckling because $R e=0.53$ is close to the value of 0.56 that limits the case of occurrence or not of jet buckling. To confirm that the results obtained with FLUID $B$ for $R e=0.53$ were due to elastic effects, an additional simulation was performed using FLUID S1 that is described in 


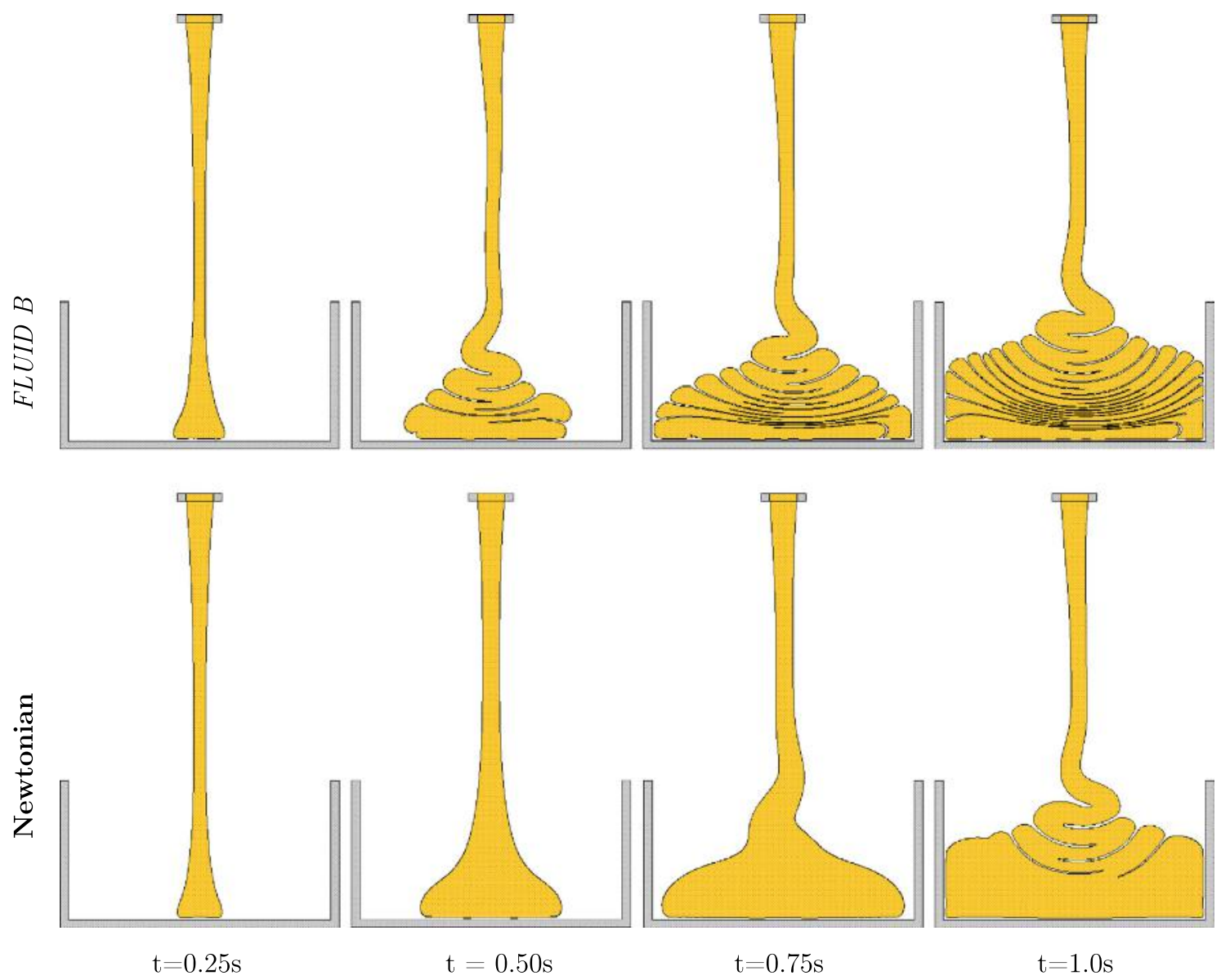

Fig. 18. Numerical simulation of impinging Newtonian and FLUID B jets with $U=0.4 \mathrm{~m} \mathrm{~s}^{-1}$. Fluid flow visualization at selected times.

Table 2. In this simulation, the data employed were: computational domain of $5 \mathrm{~cm} \times 7.2 \mathrm{~cm}$, grid size $\delta x=\delta y=1 \mathrm{~mm}$, $N=250, D=0.6 \mathrm{~cm}, U=0.28 \mathrm{~m} \mathrm{~s}^{-1}, H / D=12$ which gave $R e=0.94, W i=2.8$ and $F r=1.15$. The corresponding simulation with a Newtonian jet was also performed. Now, the conditions presented by Cruickshank and Munson for jet buckling are not satisfied and it is expected that the Newtonian jet should not undergo buckling. Indeed, as can be seen in Fig. 20, the simulation with the Newtonian jet was stable without any buckling while in the simulation with FLUID S1 the jet was unstable and presented buckling. These results confirm that elasticity modeled by the K-BKZ integral equation can lead an impinging jet to experience buckling under conditions for which a Newtonian fluid exhibits no buckling. Nevertheless, this needs to be understood with caution for two sets of reasons: (1) the previously identified stress response limitations of the K-BKZ model when subjected to uniaxial followed by biaxial extensional flow; (2) the simplified boundary conditions, especially at the inlet, relative to those of a true experiment. In regard to the first point we plot in Fig. 21 the variation of the extensional strain rate $(d v / d y)$ along the jet centre plane for several instants of $\Delta t^{*}$ following impact of the jet on the plate. Although the extensional rates are not too high at the beginning of the flow, they become negative and very intense on approaching the plate, especially at the beginning of the experiment, with the problem becoming less severe as time goes by. Only experiments or computations with constitutive equations that behave under such kinematic conditions will clarify the issue.

We observe in Figs. 17-20 that interface particles have moved to the interior of the fluid. In this case, these trapped markers are not treated as interface markers any more and may remain inside the fluid. In addition, although the boundary conditions employed in these simulations may not be appropriate, and in view of the fact that the K-BKZ model may not cope well with this problem, it is fair to say that this modeling is only a numerical test case.

\section{Concluding remarks}

The aim of this work is the numerical simulation of free surface flows governed by the integral constitutive equation K-BKZ. Initially, the governing equations together with the corresponding boundary conditions were presented, then 

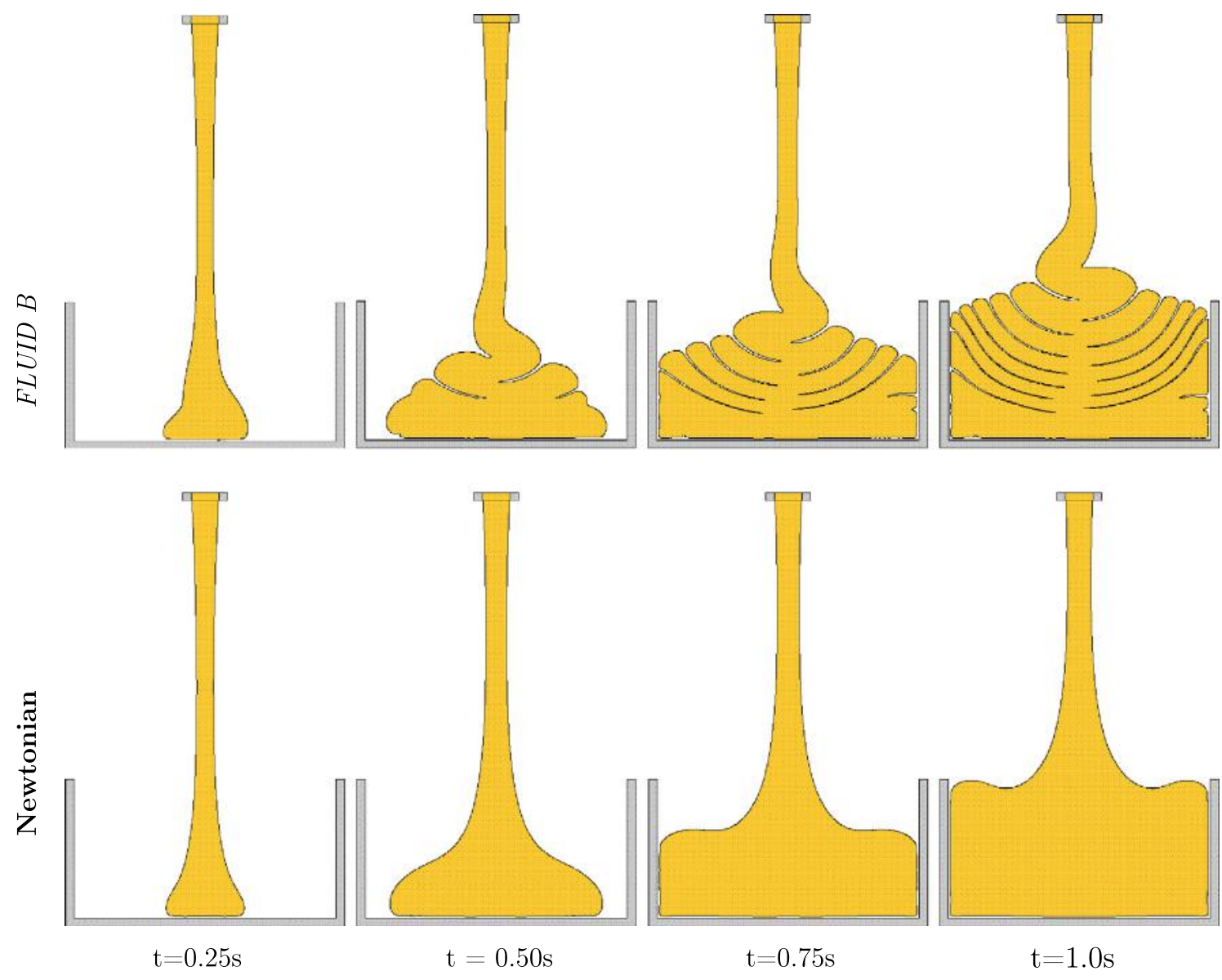

Fig. 19. Numerical simulation of impinging Newtonian and FLUID B jets with $U=0.6 \mathrm{~m} \mathrm{~s}^{-1}$. Fluid flow visualization at selected times.

two-dimensional free surface flows were considered and a numerical method for solving the basic equations by the finite difference method was described in detail. The Finger tensor was dealt with using the ideas of the deformation fields method introduced by Hulsen et al. [60]. To enhance the convergence and accuracy of the numerical method the following techniques were analyzed and implemented into a computer code:

A A new and more efficient methodology for defining the integration nodes, $t_{j}^{\prime}$, was proposed;

- A second-order difference method for calculating the Finger tensor in time was employed;

An implicit method for solving the momentum equation was introduced;

- An implicit formulation for computing the Poisson equation coupled with the corresponding equations for calculating the pressure on the moving free surface was used;

A methodology for the treatment of the fluid free surface was employed.

The finite difference computer code containing the approximations to the equations presented in Section 3 was applied to simulate flows of K-BKZ fluids. By employing mesh refinement, verification and convergence results for channel and free surface flows were provided including the verification against an analytic solution for fully developed channel flow of a 1-mode K-BKZ fluid. This analytic solution is presented in Appendix A. Furthermore, the extrudate swell of a FLUID M1 was simulated and the results were compared with those given by Mitsoulis [44]; good agreement between the two solutions was displayed.

The methodology adopted in this work was demonstrated to be capable of simulating free surface flows of fluids governed by the K-BKZ integral equation. The problem of a jet impinging on a rigid surface was simulated using fluids modeled by the K-BKZ integral constitutive equation. The fluids known as FLUID B and FLUID S1 were employed and the flows produced by the jet, for various values of the nozzle velocity, were simulated. The results showed the occurrence of the phenomenon of jet buckling over a range of conditions that were not covered by Newtonian fluids. This phenomenon is a fluid instability associated with viscous forces and compression waves and it is known that Newtonian fluids also present this kind of instability. The results obtained with Newtonian fluids are in accordance with the experimental and theoretical 


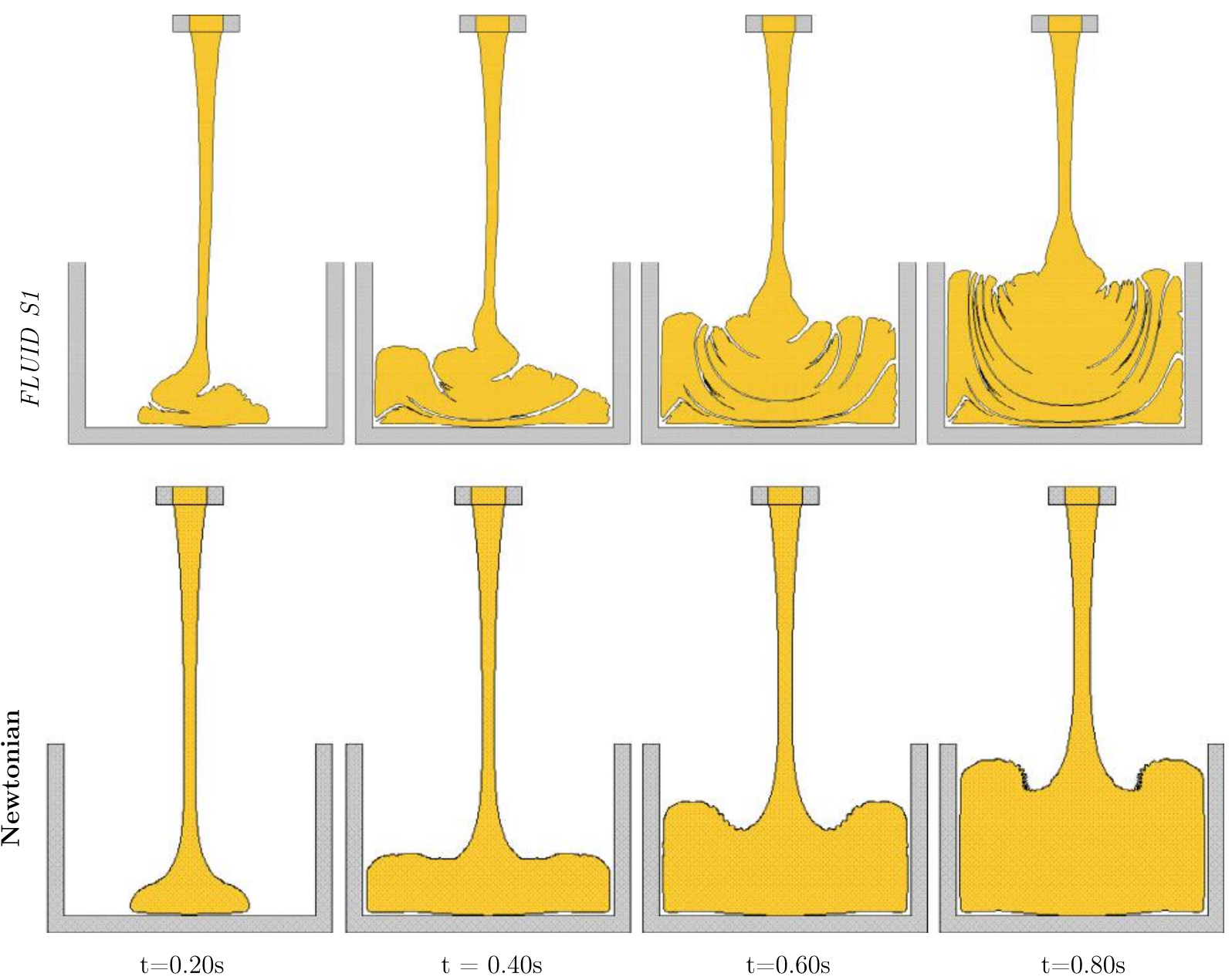

Fig. 20. Numerical simulation of impinging Newtonian and FLUID $S 1$ jets with $U=0.28 \mathrm{~m} \mathrm{~s}^{-1}$. Fluid flow visualization at selected times.

analyses of Cruickshank and Munson [65] that provide conditions for jet buckling when a Newtonian jet flow impacts onto a rigid surface. The simulations with the K-BKZ fluids showed that elasticity effects can also lead to jet buckling for flow conditions for which Newtonian fluid flows are stable.

\section{Acknowledgements}

The Brazilian authors would like to acknowledge the financial support from the funding agencies: CNPq - Conselho Nacional de Desenvolvimento Científico e Tecnológico (Grants Nos. 306280/2014-0, 301408/2009-2, 472514/2011-3, 309514/2013-4, 473589/2013-3), FAPESP - Fundação de Amparo a Pesquisa do Estado de São Paulo (Grant No. 2011/13930-0) and CAPES Grants Nos. DO/5633919, BEX 2844/10-9 and 226/09 (CAPES-FCT). This work is part of the activities developed within the CEPID-CeMEAI FAPESP project Grant No. 2013/07375-0 and also benefits from the collaboration within the framework of the University of São Paulo (Brazil) and University of Porto (Portugal) research agreements. F.T. Pinho also acknowledges funding from project MEC/MCTI/CAPES/CNPq/FAPs Grant No. 61/2011 of Programa Sem Fronteiras - Bolsas no País of the Brazilian agencies CAPES and CNPq, since it allowed several meetings of this work to take place at UFRJ/COPPE.

\section{Appendix A. Analytic solution of fully developed flow of 1-mode K-BKZ fluids}

We consider the fully-developed flow of a 1-mode K-BKZ fluid between parallel plates separated by a distance $L$. The origin of the coordinate system is at the lower plate, with $x, y$ and $z$ being the streamwise, transverse and spanwise coordinates, respectively. The transverse coordinate $y$ was normalized by $L$. We seek solutions of type

$$
u=u(y), v=0, p=p(x, y), \tau^{x y}=\tau^{x y}(y), \tau^{x x}=\tau^{x x}(y), \tau^{y y}=\tau^{y y}(y), \text { for } y \in[0,1] .
$$




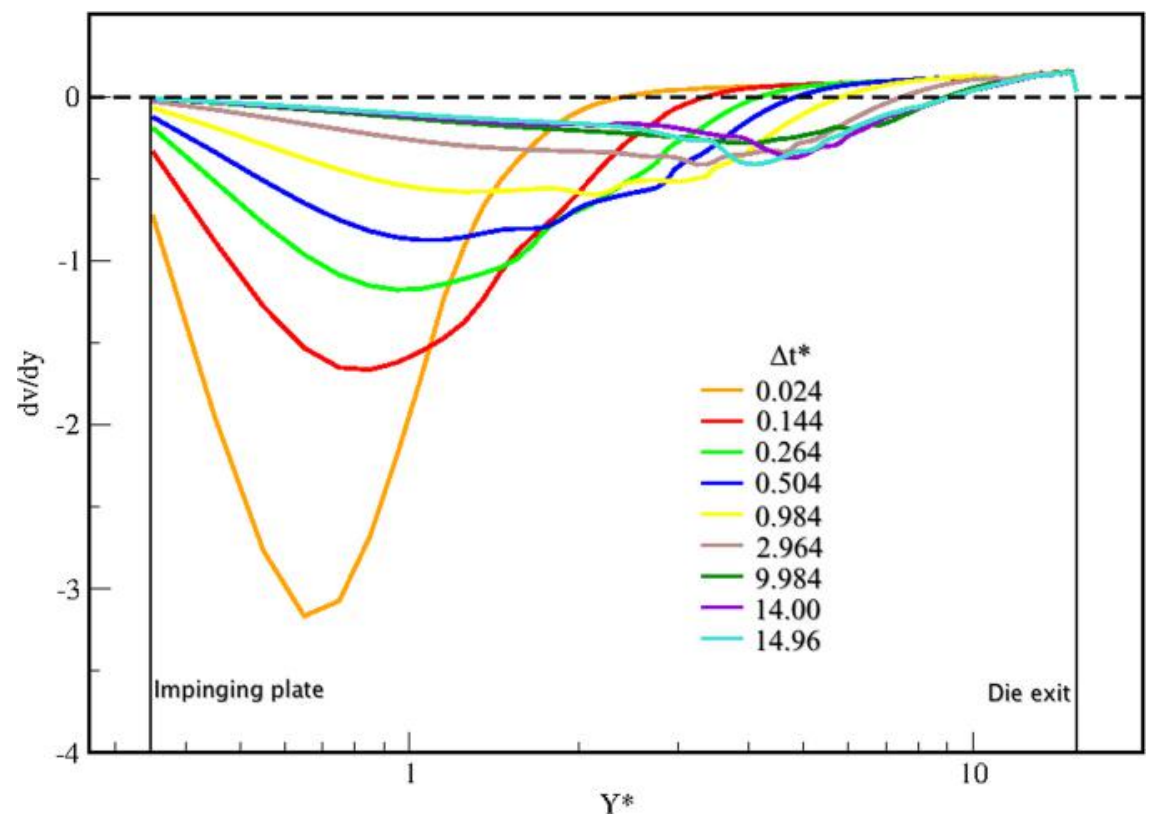

Fig. 21. Variation of extension rate along the jet centre plane. Simulation with FLUID B for $W i=8.4$ and $R e=0.53$. $\Delta t^{*}$ refers to the dimensionless time elapsed after the jet hit the impinging plate. Note that the abscissa $\left(Y^{*}=y / D\right)$ is in log-scale.

The corresponding components of the Finger tensor are given in Eq. (36) leading to the following invariants $I_{1}$ and $I_{2}$ that are required in the Papanastasiou function $H\left(I_{1}, I_{2}\right)$ in Eq. (6)

$$
I_{1}=I_{2}=3+\dot{\gamma}^{2}\left(t-t^{\prime}\right)^{2}
$$

which were normalized by $U / L$ and $\dot{\gamma}=\frac{d u}{d y}$. With these assumptions, the momentum equations reduce to

$$
\begin{aligned}
& -\frac{\partial p}{\partial x}+\frac{\partial \tau^{x y}}{\partial y}=0, \\
& -\frac{\partial p}{\partial y}+\frac{\partial \tau^{y y}}{\partial y}=0,
\end{aligned}
$$

and the equations for the components of the extra-stress tensor become

$$
\begin{aligned}
& \tau^{x y}=\frac{a_{1} \alpha}{W i} \int_{-\infty}^{t} e^{-\left(t-t^{\prime}\right) / W i} \frac{\dot{\gamma}\left(t-t^{\prime}\right)}{\alpha+\dot{\gamma}^{2}\left(t-t^{\prime}\right)^{2}} d t^{\prime}, \\
& \tau^{x x}=\frac{a_{1} \alpha}{W i} \int_{-\infty}^{t} e^{-\left(t-t^{\prime}\right) / W i} \frac{\left[1+\dot{\gamma}^{2}\left(t-t^{\prime}\right)^{2}\right]}{\alpha+\dot{\gamma}^{2}\left(t-t^{\prime}\right)^{2}} d t^{\prime}, \\
& \tau^{y y}=\frac{a_{1} \alpha}{W i} \int_{-\infty}^{t} e^{-\left(t-t^{\prime}\right) / W i} \frac{1}{\alpha+\dot{\gamma}^{2}\left(t-t^{\prime}\right)^{2}} d t^{\prime} .
\end{aligned}
$$

Equations (A.3)-(A.7) are solved as follows. Equation (A.4) gives

$$
p(x, y)=\tau^{y y}+F(x)
$$

which shows that $p(x, y)$ is a function of $x$ and $y$. Now, writing (A.3) as

$$
\frac{\partial \tau^{x y}}{\partial y}=F^{\prime}(x)
$$

where the prime denotes differentiation with respect to $x$, we see that the right-hand-side of Eq. (A.9) is a function of $y$. Consequently, $F^{\prime}$ must be a constant which we denote by $C=\frac{\partial p}{\partial x}$, hence we have

$$
\tau^{x y}(y)=C y+h(x)
$$


and since $\tau^{x y}(1 / 2)=0$ (by symmetry arguments) implies that $h(x)=-\frac{1}{2} C$ and therefore

$$
\tau^{x y}(y)=C(y-1 / 2) .
$$

Thus, equation (A.5) can be written as

$$
C(y-1 / 2)=\frac{a_{1} \alpha}{W i} \int_{-\infty}^{t} e^{-\left(t-t^{\prime}\right) / W i} \frac{\dot{\gamma}\left(t-t^{\prime}\right)}{\alpha+\dot{\gamma}^{2}\left(t-t^{\prime}\right)^{2}} d t^{\prime}
$$

and making the transformation $s=t-t^{\prime}$ yields

$$
C(y-1 / 2)=\frac{a_{1} \alpha}{W i} \int_{0}^{\infty} e^{-s / W i} \frac{\dot{\gamma} s}{\alpha+\dot{\gamma}^{2} s^{2}} d s .
$$

Now, set $s=\left(\alpha^{1 / 2} / \dot{\gamma}\right) \mathcal{T}$ to obtain

$$
C(y-1 / 2)=K \int_{0}^{\infty} e^{-\phi \mathcal{T}} \frac{\mathcal{T}}{1+\mathcal{T}^{2}} d \mathcal{T}
$$

where

$$
\phi=\frac{\alpha^{1 / 2}}{\dot{\gamma} W i}, \quad K=\frac{a_{1} \alpha^{1 / 2}}{\dot{\gamma} W i} .
$$

The integral in (A.14) can be solved by Mathematica which provides

$$
\int_{0}^{\mathcal{T}_{\infty}} e^{-\phi \mathcal{T}} \frac{\mathcal{T}}{1+\mathcal{T}^{2}} d \mathcal{T}=\frac{1}{2} e^{-i \phi}\left(-\mathrm{Ei}[i \phi]+\operatorname{Ei}\left[i \phi-\frac{\mathcal{T}_{\infty}}{W i}\right]+e^{2 i \phi}\left(-\mathrm{Ei}[-i \phi]+\operatorname{Ei}\left[-i \phi-\frac{\mathcal{T}_{\infty}}{W i}\right]\right)\right)
$$

where $i=\sqrt{-1}$, and $\operatorname{Ei}(z)$ denotes the exponential integral given by

$$
\operatorname{Ei}(z)=\int_{1}^{\infty} s^{-1} e^{-s z} d s
$$

In this case $\mathcal{T}_{\infty}$, is a value of $\mathcal{T}$ assumed to be large enough to be considered as infinity. Note that $\operatorname{Ei}(i \phi)$ is the complex conjugate of $\operatorname{Ei}(-i \phi)$, so that the result in (A.16) will be real, as is expected from (A.13). This integral can be easily solved by a high order quadrature formula. by

The constant $C$ is determined from the boundary condition at the channel entrance where a velocity $u^{i n}(y)$ is prescribed

$$
u^{\text {in }}(y)=1-4\left(y-\frac{1}{2}\right)^{2}, \quad 0 \leq y \leq 1,
$$

from which we obtain

$$
\int_{0}^{1} u^{i n}(y) d y=\frac{2}{3}
$$

and by conservation of mass $u(y)$, the solution to (A.14), must also satisfy

$$
\int_{0}^{1} u(y) d y=\frac{2}{3} .
$$

The constant $C$ must be calculated so that this equation is satisfied. At first sight, it seems that to obtain $u(y)$, equation (A.14) must be integrated with respect to $y$, subject to the boundary conditions

$$
u(0)=u(1)=0 .
$$


However, this calculation can be avoided because integration of (A.20) by parts yields

$$
\int_{0}^{1} y \dot{\gamma}(y) d y+\frac{2}{3}=0 .
$$

Therefore, the function $\dot{\gamma}$ and the unknown $C$ can be obtained by the following procedure:

\section{Procedure I}

P1. Guess a value for $C$ - an initial value can be set as the corresponding Newtonian value obtained from (A.18);

P2. Discretize the range $\left(\frac{1}{2}, 1\right]$, corresponding to a half-channel, into $N$-points, $y_{j}=\frac{1}{2}+j * \frac{1}{2 N}, j=1,2, \cdots, N$;

P3. Calculate a profile for the shear rate, $\dot{\gamma}_{j}$, using (A.14), $j=1,2, \cdots, N$;

P4. Verify if equation (A.22) is satisfied;

P5. If not, make a new guess for $C$ and perform steps P3 and P4.

After obtaining $C$ and $\dot{\gamma}\left(y_{j}\right)$ by using the Procedure I, the values of $\tau^{x x}\left(y_{j}\right)$ and $\tau^{y y}\left(y_{j}\right)$ are immediately obtained from equations (A.6) and (A.7) for which we make the change of variables $s=t-t^{\prime}$ to get

$$
\begin{aligned}
\tau^{x x}\left(y_{j}\right) & =\frac{a_{1} \alpha}{W i} \int_{0}^{\infty} e^{-s / W i} \frac{1+\dot{\gamma}\left(y_{j}\right)^{2} s^{2}}{\alpha+\dot{\gamma}\left(y_{j}\right)^{2} s^{2}} d s, \\
\tau^{y y}\left(y_{j}\right) & =\frac{a_{1} \alpha}{W i} \int_{0}^{\infty} e^{-s / W i} \frac{1}{\alpha+\dot{\gamma}\left(y_{j}\right)^{2} s^{2}} d s,
\end{aligned}
$$

and setting $s=\alpha^{1 / 2} \mathcal{T} / \dot{\gamma}\left(y_{j}\right)$, these integrals simplify to

$$
\begin{aligned}
\tau^{x x}\left(y_{j}\right) & =K \int_{0}^{\infty} e^{-\phi \mathcal{T}} \frac{1+\alpha \mathcal{T}^{2}}{1+\mathcal{T}^{2}} d \mathcal{T}, \\
\tau^{y y}\left(y_{j}\right) & =K \int_{0}^{\infty} e^{-\phi \mathcal{T}} \frac{1}{1+\mathcal{T}^{2}} d \mathcal{T},
\end{aligned}
$$

where $K$ and $\phi$ are given by equation (A.15). The integrals in (A.25) and (A.26) can be solved by Mathematica which gives

$$
\begin{aligned}
\int_{0}^{\mathcal{T}_{\infty}} e^{-\phi \mathcal{T}} \frac{1+\alpha \mathcal{T}^{2}}{1+\mathcal{T}^{2}} d \mathcal{T}= & -\frac{1}{2} e^{-i \phi-\frac{\mathcal{T}_{\infty}}{W i}}\left(-\frac{2 e^{i \phi}\left(-1+e^{\frac{\mathcal{T}_{\infty}}{\mathrm{We}}}\right) \alpha}{\phi}+i e^{\frac{\mathcal{T}_{\infty}}{\mathrm{We}}}(-1+\alpha)\right. \\
& \left.\times\left(\operatorname{Ei}[i \phi]-\mathrm{Ei}\left[i \phi-\frac{\mathcal{T}_{\infty}}{W i}\right]+e^{2 i \phi}\left(-\mathrm{Ei}[-i \phi]+\operatorname{Ei}\left[-i \phi-\frac{\mathcal{T}_{\infty}}{W i}\right]\right)\right)\right), \\
\int_{0}^{\mathcal{T}_{\infty}} e^{-\phi \mathcal{T}} \frac{1}{1+\mathcal{T}^{2}} d \mathcal{T}=- & \frac{1}{2} i e^{-i \phi}\left(\mathrm{Ei}[i \phi]-\operatorname{Ei}\left[i \phi-\frac{\mathcal{T}_{\infty}}{W i}\right]+e^{2 i \phi}\left(-\mathrm{Ei}[-i \phi]+\operatorname{Ei}\left[-i \phi-\frac{\mathcal{T}_{\infty}}{W i}\right]\right)\right) .
\end{aligned}
$$

\section{References}

[1] M.F. Tomé, A. Castelo, A.M. Afonso, M.M. Alves, F.T. Pinho, Application of the log-conformation tensor to three-dimensional time-dependent free surface flows, J. Non-Newton. Fluid Mech. 175-176 (2012) 44-54.

[2] M.F. Tomé, R.A.P. Silva, C.M. Oishi, S. McKee, Numerical solution of the upper-convected Maxwell model for three-dimensional free surface flows, Commun. Comput. Phys. 6 (2009) 367-395.

[3] G. Mompean, L. Thais, M.F. Tomé, A. Castelo, Numerical prediction of three-dimensional time-dependent viscoelastic extrudate swell using differential and algebraic models, Comput. Fluids 44 (2011) 68-78.

[4] M.F. Tomé, A. Castelo, V.G. Ferreira, S. McKee, A finite difference technique for solving the Oldroyd-B model for 3D-unsteady free surface flows, J. Non-Newton. Fluid Mech. 154 (2008) 159-192.

[5] M. Aboubacar, H. Matallah, H.R. Tamaddon-Jahromi, M.F. Webster, Highly elastic solutions for Oldroyd-B and Phan-Thien-Tanner fluids with a finite volume element method: planar contraction flows, J. Non-Newton. Fluid Mech. 103 (2002) 65-103.

[6] J.R. Clermont, M. Normandin, Numerical simulation of extrudate swell for Oldroyd-B fluid using the stream-tube analysis and a streamline approximation, J. Non-Newton. Fluid Mech. 50 (1993) 193-215. 
[7] A. Bonito, M. Picasso, M. Laso, Numerical simulation of 3D viscoelastic flows with free surfaces, J. Comput. Phys. 215 (2006) 691-716.

[8] C. Bérauto, A. Fortin, T. Coupez, Y. Demay, B. Vergnes, J.F. Agassant, A finite element method for computing the flow of multi-mode viscoelastic fluids: comparison with experiments, J. Non-Newton. Fluid Mech. 75 (1998) 1-23.

[9] G.S. Paulo, M.F. Tomé, S. McKee, A marker-and-cell approach to viscoelastic free surface flows using the PTT model, J. Non-Newton. Fluid Mech. 147 (2007) 149-174.

[10] M.F. Tomé, G.S. Paulo, M.A. Alves, F.T. Pinho, Numerical solution of the PTT constitutive equation for three-dimensional free surface flows, J. NonNewton. Fluid Mech. 165 (2010) 247-262.

[11] G.S. Paulo, C.M. Oishi, M.F. Tomé, M.A. Alves, F.T. Pinho, Numerical solution of the FENE-CR model in complex flows, J. Non-Newton. Fluid Mech. 204 (2014) 50-61.

[12] C.M. Oishi, F.P. Martins, M.F. Tomé, M.A. Alves, Numerical simulation of drop impact and jet buckling problems using the eXtended Pom-Pom model, J. Non-Newton. Fluid Mech. 169-170 (2012) 91-103.

[13] C.M. Oishi, F.P. Martins, M.F. Tomé, J.A. Cuminato, S. McKee, Numerical solution of the eXtended Pom-Pom model for viscoelastic free surface flows, J. Non-Newton. Fluid Mech. 166 (2011) 165-179.

[14] A. Goublomme, M.J. Crochet, Numerical prediction of extrudate swell of a high-density polyethylene, J. Non-Newton. Fluid Mech. 47 (1993) $281-287$.

[15] H.J. Park, D.G. Kiriakidis, E. Mitsoulis, Birefringence studies in Die Flows of an HDPE melt, J. Rheol. 36 (1992) 1563-1583.

[16] X.L. Luo, E. Mitsoulis, A numerical study of the effect of elongational viscosity on vortex growth in contraction flows of polyethylene melts, J. Rheol. 34 (1990) 309-342.

[17] B. Bernstein, E.A. Kearley, L.J. Zapas, A study of stress relaxations with finite strain, Trans. Soc. Rheol. 7 (1963) $391-410$.

[18] A. Kaye, Non-Newtonian flow in incompressible fluids, Note No. 134, College of Aeronautics, Cranfield, UK, 1962.

[19] S. Dupont, M.J. Crochet, The vortex growth of a K.B.K.Z. fluid in an abrupt contraction, J. Non-Newton. Fluid Mech. 29 (1988) 81-91.

[20] G. Barakos, E. Mitsoulis, Numerical simulation of viscoelastic flow around a cylinder using an integral constitutive equation, J. Rheol. 39 (1995) 1279.

[21] X.L. Luo, E. Mitsoulis, An efficient algorithm for strain history tracking in finite element computation of non-Newtonian fluids with integral constitutive equations, Int. J. Numer. Methods Fluids 11 (1990) 1015-1031.

[22] M. Ansari, A. Alabbas, S.G. Hatzikiriakos, E. Mitsoulis, Entry flow of polyethylene melts in tapered dies, Int. Polym. Process. 25 (2010) $287-296$.

[23] E. Mitsoulis, N.A. Malamataris, The free (open) boundary condition with integral constitutive equations, J. Non-Newton. Fluid Mech. 177-178 (2012) 97-108.

[24] P. Olley, R. Spares, P.D. Cotes, A method for implementing time-integral constitutive equations in commercial CFD packages, J. Non-Newton. Fluid Mech. 86 (1999) 337-357.

[25] M.F. Tomé, M.S.B. Araujo, M.A. Alves, F.T. Pinho, Numerical simulation of viscoelastic flows using integral constitutive equations: a finite difference approach, J. Comput. Phys. 227 (2008) 4207-4243.

[26] K. Osaki, S. Kimura, M. Kurata, Relaxation of shear and normal stresses in double-step shear deformations for a polystyrene solution. A test of the Doi-Edwards theory for polymer rheology, J. Rheol. 25 (1981) 549-562.

[27] J.K. Nielsen, H.K. Rasmussen, Reversed extension flow, J. Non-Newton. Fluid Mech. 155 (2008) 15-19.

[28] Q. Huang, H.K. Rasmussen, A.L. Skov, O. Hassager, Stress relaxation and reversed flow of low-density polyethylene melts following uniaxal extension, J. Rheol. 56 (2012) 1535-1554.

[29] R.G. Larson, The Structure and Rheology of Complex Fluids, Oxford University Press, New York, 1999.

[30] A.C. Papanastasiou, L.E. Scriven, C.W. Macosko, An integral constitutive equation for mixed flows: viscoelastic characterization, J. Rheol. 27 (1983) $387-410$

[31] E. Mitsoulis, 50 years of the K-BKZ constitutive relation for polymers, Polym. Sci. 2013 (2013) 952379.

[32] D.W. Mead, R.G. Larson, M. Doi, A molecular theory for fast flows of entangled polymers, Macromolecules 31 (1998) $7895-7914$

[33] G. Ianniruberto, G. Marrucci, A simple constitutive equation for entangled polymers with chain stretch, J. Rheol. 45 (2001) 1305-1318.

[34] M.H. Wagner, S. Kheirandish, O. Hassager, Quantitative prediction of transient and steady-state elongation viscosity of nearly monodisperse polystyrene melts, J. Rheol. 49 (2005) 1317-1327.

[35] O. Hassager, C. Bisgaard, A Lagrangian finite element method for the simulation of flow of non-Newtonian liquids, J. Non-Newton. Fluid Mech. 12 (1983) 153-164.

[36] H.K. Rasmussen, O. Hassager, Three-dimensional simulations of viscoelastic instability in polymeric filaments, J. Non-Newton. Fluid Mech. 82 (1999) $189-202$.

[37] E.A.J.F. Peters, M.A. Hulsen, B.H.A.A. van den Brule, Instationary Eulerian viscoelastic flow simulations using time separable Rivlin-Sawyers constitutive equations, J. Non-Newton. Fluid Mech. 89 (2000) 209-228.

[38] E.A.J.F. Peters, A.P.G. van Heel, M.A. Hulsen, B.H.A.A. van den Brule, Generalization of the deformation field method to simulate advanced reptation models in complex flow, J. Rheol. 44 (2000) 811-829.

[39] P. Wapperom, R. Keunings, G. Ianniruberto, Prediction of rheometrical and complex flows of entangled linear polymers using the double-convectionreptation model with chain stretch, J. Rheol. 47 (2003) 247-265.

[40] G.H. McKinley, T. Sridhar, Filament-stretching rheometry of complex fluids, Annu. Rev. Fluid Mech. 34 (2002) $375-415$.

[41] M.I. Kolte, H.K. Rasmussen, O. Hassager, Transient filament stretching rheometer II: numerical simulation, Rheol. Acta 36 (1997) $285-302$.

[42] A. Bach, H.K. Rasmussen, P. Longin, O. Hassager, Growth of non-axisymmetric disturbances of the free surface in the filament stretching rheometer: experiments and simulation, J. Non-Newton. Fluid Mech. 108 (2002) 163-186.

[43] M.S. Chai, Y.L. Yeow, Modelling of fluid M1 using multiple-relaxation-time constitutive equations, J. Non-Newton. Fluid Mech. 35 (1990) $459-470$.

[44] E. Mitsoulis, Extrudate swell of Boger fluids, J. Non-Newton. Fluid Mech. 165 (2010) 812-824.

[45] X.L. Luo, E. Mitsoulis, Memory phenomena in extrudate swell simulations for annular dies, J. Rheol. 33 (1989) $1307-1327$.

[46] A. Goublomme, B. Draily, M.J. Crochet, Numerical prediction of extrudate swell of a high-density polyethylene: further results, J. Non-Newton. Fluid Mech. 44 (1992) 171-195.

[47] V. Ganvir, A. Lele, R. Thaokar, B.P. Gautham, Prediction of extrudate swell in polymer melt extrusion using an arbitrary Lagrangian Eulerian (ALE) based finite element method, J. Non-Newton. Fluid Mech. 156 (2009) 21-28.

[48] R. Ahmed, R.F. Liang, M.R. Mackley, The experimental observation and numerical prediction of planar entry flow and die swell for molten polyethylenes, J. Non-Newton. Fluid Mech. 59 (1995) 129-153.

[49] O. Hassager, M.I. Kolte, M. Renardy, Failure and nonfailure of fluid filaments in extension, J. Non-Newton. Fluid Mech. 76 (1998) $137-151$.

[50] H.K. Rasmussen, Time-dependent finite-element method for the simulation of three-dimensional viscoelastic flow with integral models, J. Non-Newton. Fluid Mech. 84 (1999) 217-232.

[51] J.M.R. Marín, H.K. Rasmussen, Lagrangian finite-element method for the simulation of K-BKZ fluids with third order accuracy, J. Non-Newton. Fluid Mech. 156 (2009) 177-188.

[52] O. Hassager, J.M.N. Marin, K. Yu, H.K. Rasmussen, Polymeric liquids in extension: fluid mechanics or rheometry, Rheol. Acta 49 (2010) 543-554.

[53] R.I. Tanner, From A to (BK)Z in constitutive relations, J. Rheol. 32 (1988) 673-702.

[54] D. Rajagopalan, R.C. Armstrong, R.A. Brown, Finite element methods for calculation of steady viscoelastic flow using constitutive equations with Newtonian viscosity, J. Non-Newton. Fluid Mech. 36 (1990) 159-192. 
[55] G.K. Batchelor, An Introduction of Fluid Dynamics, Cambridge University Press, Cambridge, 1967.

[56] A.A. Amsden, F.H. Harlow, A simplified MAC technique for incompressible fluid flow calculations, J. Comput. Phys. 6 (1970) $332-335$.

[57] F.H. Harlow, J.E. Welch, Numerical calculation of time-dependent viscous incompressible flow of fluid with free surface, Phys. Fluids 8 (1965) 2182-2189.

[58] M.F. Tomé, A. Castelo, J. Murakami, J.A. Cuminato, R. Minghin, M.C.F. Oliveira, S. McKee, Numerical simulation of axisymmetric free surface flows, J. Comput. Phys. 157 (2000) 441-472.

[59] M.F. Tomé, S. McKee, GENSMAC: a computational marker-and-cell method for free surface flows in general domains, J. Comput. Phys. 110 (1994) $171-186$.

[60] M.A. Hulsen, E.A.J.F. Peters, B.H.A.A. van den Brule, A new approach to the deformation fields method for solving complex flows using integral constitutive equations, J. Non-Newton. Fluid Mech. 98 (2001) 201-221.

[61] M.F. Tomé, B. Duffy, S. McKee, A numerical technique for solving unsteady non-Newtonian free surface flows, J. Non-Newton. Fluid Mech. 62 (1996) $9-34$.

[62] M. Alves, P. Oliveira, F.T. Pinho, A convergent and universally bounded interpolation scheme for the treatment of advection, Int. J. Numer. Methods Fluids 41 (2003) 47-75.

[63] L.M. Quinzani, G.H. McKinley, R.A. Brown, R.C. Armstrong, Modeling the rheology of polyisobutylene solutions, J. Rheol. 34 (1990) 705-748.

[64] R.I. Tanner, Engineering Rheology, 2nd ed., Oxford University Press, Oxford, UK, 2000.

[65] J.O. Cruickshank, B.R. Munson, Viscous fluid buckling of plane and axisymmetric jets, J. Fluid Mech. 113 (1981) $221-239$.

[66] M.F. Tomé, S. McKee, L. Barratt, D.A. Jarvis, A.J. Patrick, An experimental and numerical investigation of container filling with viscous liquids, Int. J. Numer. Methods Fluids 31 (1999) 1333-1351.

[67] N.M. Ribe, A general theory of the dynamics of thin viscous jets, J. Fluid Mech. 457 (2002) 255-283.

[68] M.F. Tomé, A. Castelo, J.A. Cuminato, N. Mangiavacchi, S. McKee, GENSMAC3D: a numerical method for solving unsteady three-dimensional free surface flows, Int. J. Numer. Methods Fluids 37 (2001) 747-796.

[69] X. Xu, J. Ouyang, T. Jiang, Q. Li, Numerical simulation of 3D-unsteady viscoelastic free surface flows by improved smoothed particle hydrodynamics method, J. Non-Newton. Fluid Mech. 177-178 (2012) 109-120.

[70] M.F. Tomé, A. Castelo, M. Nóbrega, O. Carneiro, G.S. Paulo, F.T. Pereira, Numerical and experimental investigations of three-dimensional container filling with Newtonian viscous fluids, Comput. Fluids 90 (2014) 172-185. 